\title{
Espacio del producto y cambio estructural: un enfoque latinoamericano y una aplicación al caso uruguayo *
}

\author{
Álvaro Brunini \\ Sebastián Fleitas * \\ Guzmán Ourens **
}

\begin{abstract}
Resumen
Este artículo se inserta en el debate al respecto de los determinantes de la convergencia de los ingresos de los países y parte de la idea de que la estructura productiva es uno de los fundamentales para su explicación. En este sentido, se realiza un estudio comparativo sobre las potencialidades para el cambio estructural entre Uruguay y otros países que presentan una especialización similar a éste. Para esto se reinterpreta el Espacio del Producto creado por Hausmann y otros investigadores de la Universidad de Harvard, desde la visión de los trabajos más recientes de la CEPAL, que identifican en las capacidades tecnológicas una de los principales determinantes de las potencialidades de las estructuras productivas. La principal conclusión del trabajo es que Uruguay presenta diferentes potencialidades para el cambio estructural en comparación a un conjunto de países con estructuras productivas que, a priori, podían considerarse "similares" a la suya.
\end{abstract}

Palabras clave: Convergencia; Cambio estructural; Capacidades tecnológicas; Espacio del producto.

\begin{abstract}
Product space and structural change: a Latin American approach and an application to the Uruguayan case

This article immerses into the debate about the determinants of countries income level convergence, and states that the productive structure of a country is one of the most important of such determinants. A comparative study is performed, testing the potentiality for structural change for Uruguay and nine other countries with a commercial specialization that could be considered similar between them. In order to do this, the article proposes a new interpretation of the Product Space created by Hausmann and other Harvard University researchers, based upon the theoretical framework of the latest works of the ECLALC, which point to technological capabilities as one of the most important determinants of the potentialities of productive structures. The article's main conclusion is that Uruguay shows different potentialities for structural change from those countries with a similar specialization.
\end{abstract}

Key words: Convergence; Structural change; Technological capabilities; Product space.

JEL F19, O14, O33, O57.

\section{Introducción}

Uruguay ha cursado una senda de crecimiento pobre en las últimas cinco décadas, generando un marcado proceso de divergencia de ingresos en relación a

\footnotetext{
* Trabalho recebido em 14 de outubro de 2010 e aprovado em 15 de março de 2012.

** Docentes de la Universidad de la República (UdelaR), Montevideo, Uruguay. E-mails: abrunini@iecon.ccee.edu.uy; sefle@iecon.ccee.edu.uy; guzman@decon.edu.uy, respectivamente.
} 
los países ricos lo que limitó el desarrollo del país. Compartiendo este diagnóstico, un conjunto de trabajos ha señalado que la estructura productiva de Uruguay podría ser una de las causas del escaso crecimiento de su economía a largo plazo (Bittencourt, 2003, 2006; Bértola, 2000; Álvarez et al., 2007; PNUD, 2008).

Estos estudios recuperan en parte el legado de la Comisión Económica para América Latina y el Caribe (CEPAL), el cual tiene una larga tradición en afirmar que el tipo de la estructura productiva afecta al crecimiento a largo plazo. De forma simplificada, puede decirse que los vínculos entre estructura productiva y crecimiento vienen dados porque el crecimiento depende de la acumulación de conocimientos y éstos se encuentran imbricados en la estructura productiva.

En la CEPAL (2007) se sostiene que si se adopta una concepción de estructura productiva basada en las acumulaciones de capacidades tecnológicas, las diferencias entre las estructuras productivas pueden ser captadas de mejor manera. Esta tradición se articula además con las teorías evolucionistas del cambio técnico y el crecimiento, como las desarrolladas por Dosi (1982), Nelson y Winter (1982), entre otros.

Las teorías del crecimiento endógeno han incorporado los efectos de las estructuras productivas en el crecimiento, sosteniendo que, tanto el capital humano (ligado a la educación y a la experiencia) como el conocimiento en general (capital de conocimiento), poseen la propiedad de crear nuevo conocimiento. De esta forma, se ingresa a la consideración de rendimientos marginales crecientes a escala, cuestionando los fundamentos de las teorías neoclásicas tradicionales que parten de la consideración del supuesto de rendimientos marginales decrecientes. A partir de los años ochenta del siglo $\mathrm{XX}$, las nuevas teorías del crecimiento endógeno, presentan modelos con sectores intensivos en $\mathrm{I}+\mathrm{D}$ y con cambio estructural, que permiten el crecimiento de las economías a largo plazo. El cambio estructural se incorpora en los modelos a través de la creación de nuevos bienes de capital, el aumento en la división del trabajo o la mayor calidad de los bienes (Romer, 1986; Aghion y Howit, 1992; Grossman y Helpman, 1991). Así, diferentes estructuras productivas dan lugar a diferentes procesos de crecimiento debido a la generación de externalidades tecnológicas (derrames de tecnologías y retornos crecientes), de indivisibilidades y complementariedades.

En los últimos años la corriente neoclásica comenzó a prestar mayor atención a estos temas debido, entre otras cosas, a los trabajos realizados por un grupo de trabajo en la Universidad de Harvard, liderados por Ricardo Hausmann. Basados en un acercamiento fuertemente empírico, estos autores sostienen que los países que han logrado alcanzar la producción de bienes con mayor productividad promedio a nivel mundial, tienden a presentar un mejor desempeño en términos de crecimiento del PBI per cápita. De esta forma, la clave del crecimiento está en la 
capacidad que tengan los países para producir bienes de alta productividad (Hausmann et al., 2005). Los autores encuentran que dicha capacidad puede ser aproximada al tipo de bienes que los países actualmente exportan, con ventajas comparativas.

El presente trabajo de investigación utiliza como marco teórico los planteos de la CEPAL para reinterpretar el herramental propuesto por Hausmann y otros. Se entiende que la importancia dada a la estructura productiva para los procesos de crecimiento y desarrollo, es un elemento común tanto en los planteos de la CEPAL (Prebisch, Fajnzylber, y los trabajos más recientes) como en los planteos del grupo liderado por Hausmann. Se recupera así una rica tradición teórica (cuyo foco ha sido la relación entre tecnología, estructura productiva y crecimiento desde comienzos de los cincuenta), para discutir evidencias y metodologías empíricas desarrolladas de forma más reciente, ya que, se entiende que la tradición teórica estructuralista permite ampliar sustancialmente las posibilidades analíticas de la nueva metodología.

Se pretende abordar la siguiente pregunta: ¿Cuál es el potencial para la transformación productiva de distintos países, considerando las capacidades existentes y sus patrones de especialización, desde una perspectiva comparada? Al adoptar como marco teórico los planteos de la CEPAL, se entiende que el nexo teórico entre estructura productiva y convergencia de ingresos per cápita, se encuentra en las capacidades tecnológicas que posee un país. Estas son las que definen su potencialidad para transformar su estructura productiva de modo a superar la heterogeneidad estructural existente, que se percibe como una de las últimas causas de la divergencia de ingresos.

La hipótesis de partida es que países de similar especialización pueden mostrar capacidades productivas muy distintas, con efectos importantes sobre el potencial de crecimiento.

\section{Marco teórico}

Un conjunto de trabajos (CEPAL, 2000, 2002, 2004, 2007, 2008; Cimoli, 2005; Cimoli, et al., 2008) elaborados en el seno de la CEPAL durante la última década, indican que esta institución desarrolló durante estos años una reformulación del análisis de los procesos de crecimiento y su vinculación con el cambio estructural. Dichas reformulaciones son derivadas del enfoque tradicional de la CEPAL que identifica el cambio de la estructura productiva, provocado por las incorporaciones de cambio técnico, como el único capaz de revertir la tendencia divergente en la riqueza por habitante entre centros y periferia. 


\subsection{Los canales de trasmisión desde la estructura productiva al crecimiento}

En Cimoli (2005) y Porcile et al. (2006) se desarrollan los dos canales por los cuales la especialización productiva afecta el crecimiento a largo plazo.

Por un lado, el "canal schumpeteriano" se encuentra ligado a las asimetrías tecnológicas que condicionan la oferta de bienes de los países. Cuando un país presenta una brecha tecnológica muy elevada sólo podrá producir un número muy reducido de bienes, los cuales presentarán baja productividad relativa y menor intensidad tecnológica. A su vez, como los sectores con baja intensidad tecnológica presentan un menor crecimiento relativo de la productividad, este tipo de especialización determinará menores potencialidades de aumento de la productividad a largo plazo a través del aprendizaje. Así el crecimiento de estos países sería menor largo plazo que el crecimiento de los países centrales.

Por su parte, el "canal keynesiano" vinculado a la demanda externa, está dado por la correlación entre la intensidad tecnológica de los bienes y el dinamismo de la demanda internacional. Esta correlación implica que los países de la periferia se encuentran más expuestos a crisis recurrentes de la balanza de pagos, ya que al tener una baja elasticidad-ingreso de las exportaciones y una elevada elasticidad-ingreso de las importaciones, enfrentarán crisis de deuda y fuertes ajustes externos toda vez que emprendan un proceso de crecimiento sostenido.

La conjunción de estos canales reduce el crecimiento efectivo de las economías de la periferia e impide el pleno aprovechamiento de las oportunidades asociadas a los retornos crecientes, el aprendizaje en la inversión y la producción. Así se produce la divergencia de ingresos internacionales de las economías de la periferia respecto a las economías del centro, al tiempo que aumenta la brecha de aprendizaje y de las capacidades tecnológicas.

\subsection{Capacidades tecnológicas, aprendizaje y potencialidades para el cambio estructural}

En los trabajos más recientes de la CEPAL, los procesos de transformación de la estructura productiva se entienden en el marco de la microeconomía de la teoría evolucionista. La corriente evolucionista (o neo-schupeteriana) enfoca su concepción del progreso técnico en las aplicaciones del mismo al proceso productivo.

De acuerdo con esta corriente, el proceso productivo no se limita solamente a las interacciones dentro de la empresa, ya que las empresas se relacionan entre ellas y generan así redes, más allá de los sectores al que pertenece. El conjunto de redes y sectores, y la forma en que se interrelacionan, es lo que conforma, según esta corriente, la estructura productiva. 
A su vez, como se plantea en Burgueño y Pittaluga (1994), la incorporación de tecnología (o sea, el proceso de aprendizaje) tiene tres características fundamentales. Por un lado, el conocimiento tiene un componente tácito que se vincula a las capacidades no codificables ni transmisibles que las empresas tienen de hacer las cosas. En segundo lugar, establecen que la tecnología presenta una elevada capacidad de ser acumulada, por lo que las nuevas innovaciones que se realicen dependerán fuertemente de la trayectoria tecnológica que la empresa haya emprendido. Por último, afirman que los procesos de innovación son en general discontinuos, dado que cada cambio de paradigma tecnológico (que se asocia con la aparición de innovaciones radicales), implica un salto en el límite superior de la evolución tecnológica.

Estas características determinan que las diferentes empresas posean distintas aptitudes para explorar las oportunidades tecnológicas y aplicarlas a procesos y productos específicos. Si bien hay una parte de las innovaciones transferible a través de publicaciones o incluso a través de externalidades tecnológicas, existe una parte importante que no resulta transferible y que torna específicos los procesos, por lo que cada empresa seguirá su propia trayectoria tecnológica. Es de esta forma que el conocimiento se imbrica en la estructura productiva: ésta sintetizará la conjunción de los diversos procesos de aprendizaje realizados por las empresas en las actividades de producción e intercambio (López, 1996, p. 14).

La corriente evolucionista afirma que, dado el carácter tácito y acumulativo de las innovaciones, tanto las oportunidades tecnológicas aprovechadas como las potenciales tienen un fuerte carácter sectorial, lo que deriva en la existencia de una fuerte diferenciación intersectorial de las oportunidades tecnológicas. Pero, a su vez, el enfoque supera la mirada sectorial al realzar la importancia de las redes de innovación en el proceso de aprendizaje e incorporación de innovaciones. Así, cuanto más desarrolladas estén las redes mayores serán las capacidades tecnológicas de la estructura productiva que integran. Esto se engloba en el concepto del Sistema Nacional de Innovación (SNI), el cual pretende resaltar el papel protagónico de las empresas y su entorno (los centros de investigación, las universidades) en la aparición de innovaciones a nivel de toda la economía. En este marco, el conjunto de posibilidades de producción de un país incluye "todas las formas de producción que pueden ser desarrolladas a partir del estado actual del conocimiento tecnológico, con independencia de si fueron aplicadas o no" (Burgueño y Pittaluga, 1994, p. 2). 


\section{Un nuevo marco analítico para estudiar el cambio estructural}

\subsection{El Espacio del Producto a la luz de las capacidades tecnológicas}

Hausmann y Klinger (2006) se preguntan qué gobierna el patrón de la transformación estructural o, centrando el interés en el patrón de especialización productiva, qué determina la evolución de las ventajas comparativas reveladas de un país entre los bienes. Los autores plantean que los cambios en las ventajas comparativas reveladas de los países son gobernados por patrones de "parentesco" entre los bienes. Las nuevas producciones tienden a surgir en bienes que tienen mayores parentescos con los bienes que ya se producen. Para construir lo que denominan el "Espacio del Producto" utilizan dos indicadores fundamentales.

Definen la ventaja comparativa revelada $(R C A)$ en el sentido de Balassa (1986) como:

$$
R C A_{c, i}=\frac{\frac{e(c, i)}{\sum_{i} e(c, i)}}{\frac{\sum_{c} e(c, i)}{\sum_{c, i} e(c, i)}} \geq 1
$$

denotando $e$ a las exportaciones y con el subíndice $c$ los países y con $i$ a los bienes.

A partir de ello, la proximidad (que mide el parentesco o similitud) entre dos bienes $j$ e $i$, se define como el mínimo de la probabilidad condicionada en que los países que presentan ventaja comparativa revelada en $i$ presenten ventaja comparativa revelada en $j$ y de que los países que tienen ventaja comparativa revelada en $j$ presentan ventaja comparativa en $i$. Formalmente:

$$
\text { (2) } \phi_{i j}=\min \left\{P\left(R C A_{e_{i}} / R C A_{e_{j}}\right), P\left(R C A_{e_{j}} / R C A_{e_{i}}\right)\right\}
$$

A partir de esta medida de proximidad entre bienes es posible construir una matriz de proximidades entre los bienes que configura el Espacio del Producto. Para construir la matriz de las proximidades se toman en cuenta los bienes exportados en el período 1998-2000. Tal como se realiza en Hidalgo et al. (2007), el Espacio del Producto se puede graficar calculando el "árbol más expandido" (maximum spanning tree), que incluye los 774 vínculos que existen entre los 775 bienes tomados en cuenta, que maximizan la proximidad agregada del árbol, y superponiendo a él todos los vínculos con una proximidad mayor a 0,55. 
Figura 1

Representación del espacio del producto

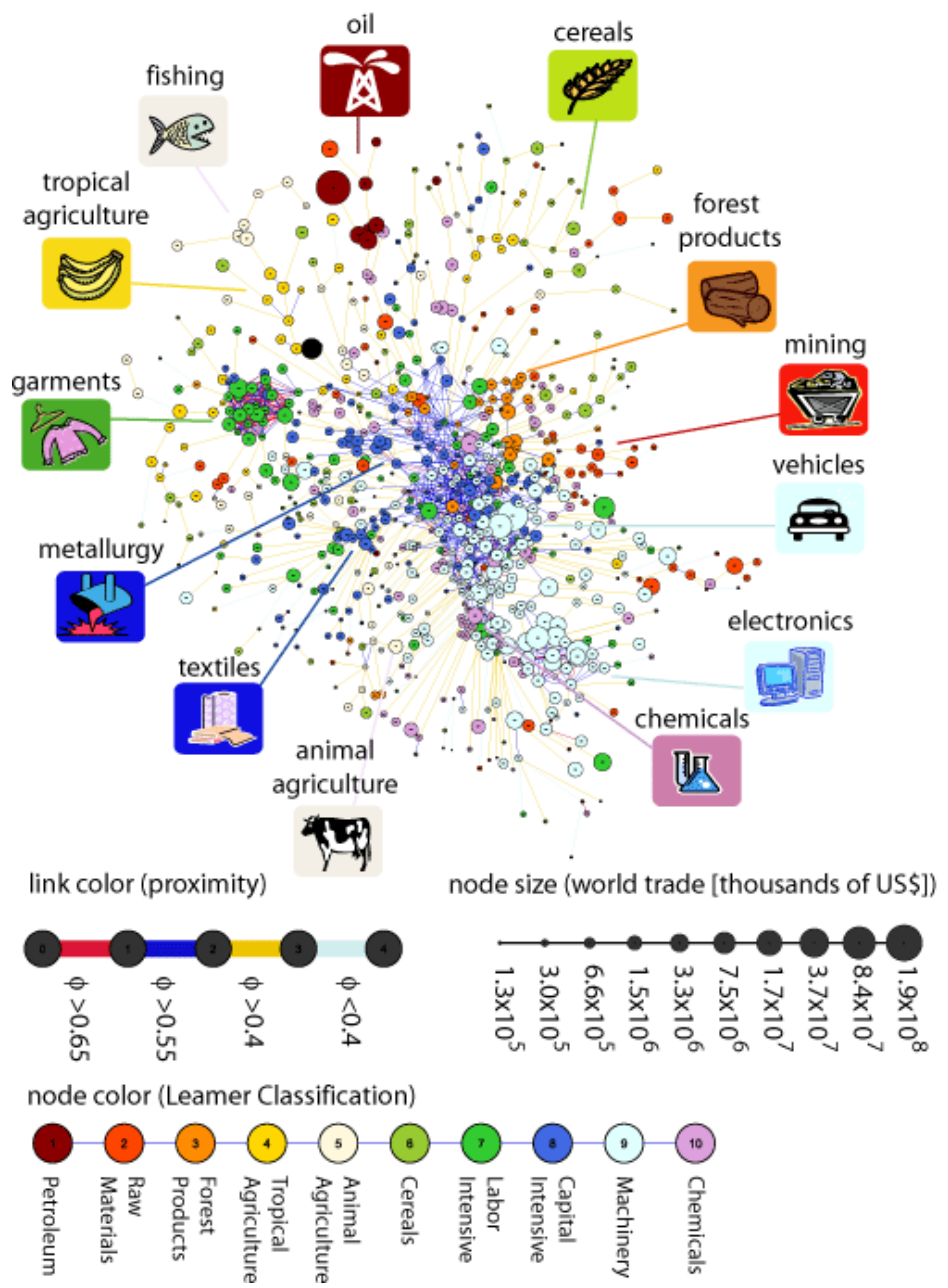

Tomado de Hidalgo et al. (2007).

Nota: El Espacio del Producto posee referencias en colores. Los colores de los nodos representan la clasificación de Leamer (1984). El tamaño de los nodos es proporcional al valor del comercio mundial de cada bien. Por último, los colores en los enlaces indican un rango de proximidad entre los bienes: el color celeste es para los bienes menos próximos y el color rojo para los más próximos. La versión en colores puede visualizarse en http://www.chidalgo.com/productspace/network.htm.

Los colores que adquieren los nodos se refieren a las diferentes categorías de la clasificación de Leamer (1984) como se establece en la figura. El tamaño de los nodos está relacionado con el volumen del comercio mundial de cada bien. Por último, los colores en los enlaces indican el rango de los valores de la proximidad 
entre los bienes, el color celeste es para los bienes menos próximos y el color rojo para los bienes más próximos.

La parte más densa del Espacio del Producto, o sea donde se encuentran un conjunto más amplio de bienes próximos entre sí, tiende a ser dominada por bienes manufacturados mientras que la parte menos densa tiende incluir bienes agrícolas no procesados.

Los autores desarrollan además, un conjunto de indicadores para analizar las características de los bienes en este Espacio del Producto. Hausmann et al. (2005) construyen la variable $P R O D Y$, utilizada como una aproximación a la productividad asociada a cada bien, que adjudica un nivel del producto per cápita a cada bien, a partir de la sumatoria del producto per cápita de cada país exportador, ponderado por el cociente entre la participación de las exportaciones de ese producto, sobre el total de las exportaciones de ese país y la suma de las participaciones de ese producto en la canasta de cada país exportador. Formalmente,

$$
\text { (3) } P R O D Y_{i}=\sum_{c} \frac{\left(e_{c i} / E c\right)}{\sum_{c}\left(e c i / E_{c}\right)} Y_{c}
$$

donde $Y_{c}$ es el PIB per cápita del país $c, e_{\underline{j c}}$ representa las exportaciones del bien $i$ por el país $c$ y $E_{c}$ representa el total de exportaciones del país $c$.

Posteriormente, se construye un indicador del nivel de sofisticación de la canasta de exportaciones a partir de la ponderación del PRODY de cada bien exportado por la participación de ese bien en la canasta de exportaciones del país. Formalmente,

$$
\text { (4) } E X P Y_{c}=\sum_{i}\left(\frac{e_{c i}}{E_{i}}\right) \cdot P R O D Y_{i}
$$

donde el ponderador es el peso del bien $i$, en la canasta de exportaciones del país $c$.

En diferentes trabajos, los autores encuentran que el EXPY tiene un efecto positivo sobre el crecimiento (al igual que las variables habituales de convergencia condicional) y que la significación de este efecto es intrínseca al cambio estructural y no viene dada porque la variable del cambio estructural recoja indirectamente efectos de la dotación factorial ni de la calidad institucional de los países, que fueron controladas.

Si como se plantea anteriormente las proximidades entre pares de bienes son importantes, entonces la probabilidad de que un país exporte un bien en el 
futuro con ventajas comparativas dependerá de la dificultad o facilidad que el país tenga para readaptar las capacidades existentes para la producción de un nuevo bien. En este sentido, la proximidad que tenga el nuevo bien respecto a alguno o algunos de los bienes pertenecientes a la canasta actual de exportaciones es relevante, ya que ésta canasta contiene las capacidades existentes en el país. Por esto calculan la densidad, definida como la proporción de la distancia de un bien hacia cada uno de los bienes de la actual canasta de exportación en el total de las distancias de ese bien, midiendo entonces la concentración de la producción actual de un país alrededor de cualquier bien. Formalmente,

$$
\text { (5) densidad } i, c, t=\left[\frac{\sum_{k} \phi_{i, k, t} \cdot x_{c, k, t}}{\sum_{k} \phi_{i, k, t}}\right]
$$

donde el subíndice $t$ se refiere al año, $i$ se refiere al bien para el cual se calcula la densidad, $c$ representa al país y $x_{c, k, t}$ es una variable indicadora, que toma valor uno si el país tiene ventaja comparativa revelada en ese bien y cero en otro caso, el subíndice $k$ se refiere a los bienes de la canasta de exportación que se producen con ventajas comparativas reveladas.

La densidad varía entre 0 y 1 , valores cercanos a 1 significan que el país ha desarrollado ventajas comparativas reveladas en una gran cantidad de bienes cercanos al bien que se está analizando, existiendo, de esta forma, una mayor probabilidad de que en el futuro se exporte ese bien. Si este análisis fuera correcto, entonces debería existir una importante correlación entre la densidad en un período y el surgimiento de la producción de esos nuevos bienes en los siguientes períodos. En Hausmann y Klinger (2007) se testea esta hipótesis en donde los autores llegan a la conclusión de que la densidad es importante para el cambio estructural, encuentran que el incremento de un desvío estándar en la densidad lleva a un incremento del $6 \%$ en la probabilidad de moverse hacia el nuevo bien.

Con el único fin de lograr resultados más fácilmente legibles, en este trabajo se optó por utilizar el inverso de la densidad como variable, a la cual se denominó lejanía, y que formalmente se define como:

$$
\text { (6) } \text { leianía }_{i, c, t}=(-1) * \log \left(\text { densidad }_{i, c, t}\right)
$$

De esta manera un bien con una lejanía reducida sería aquel para el cual el país encuentra relativamente más sencilla su producción dadas las acumulaciones tecnológicas que posea. 
Para analizar la importancia estratégica que presenta la canasta de exportaciones de ese país y, más aún, cada uno de los bienes que exporta mide en qué grado la canasta actual de exportaciones del país está conectada con nuevas posibilidades productivas (siempre analizando aquellos bienes que el país exporta con ventajas comparativas). Para ello, se utiliza un indicador que considera el nivel del $P R O D Y$ de los bienes no exportados ponderado por la densidad con la canasta de exportación, al cual se lo denomina open forest:

$$
\text { (7) } \text { open_forest }_{c, t}=\sum_{i} \sum_{j}\left[\frac{\phi_{i, j, t}}{\sum_{i} \phi_{i, j, t}} \cdot\left(1-x_{j, c, t}\right) \cdot x_{i, c, t} \cdot P R O D Y_{j, t}\right]
$$

Los autores encuentran que el open forest es un buen predictor del EXPY. De acuerdo a Hausmann y Klinger (2006) los cambios en el open forest conducen a un consiguiente incremento del EXPY, o sea, que la proximidad de las nuevas oportunidades medidas por el open forest, determina de manera significativa, el crecimiento del EXPY.

A su vez, se define un valor estratégico para aquellos bienes no exportados con ventajas comparativas reveladas como la contribución que éste haría al open forest del país en el caso de que pasara a ser producido con ventaja comparativa revelada y que se continuara exportando con ventajas comparativas reveladas el conjunto de bienes que el país ya producía. Se considera que el indicador recoge el valor estratégico del bien ya que mide el valor de los bienes a los cuales es posible acceder a partir de comenzar a exportar con ventajas comparativas reveladas en ese bien. Formalmente,

$$
\text { (8) valor_estratégic }{ }_{b, c, t}=\sum_{j}\left[\frac{\phi_{b, j, t}}{\sum_{i} \phi_{i, j, t}} \cdot\left(1-x_{j, c, t}\right) \cdot \text { PRODY }_{j, t}\right]
$$

donde el subíndice $b$ se refiere al bien para el cual se realiza el cálculo y que no se exporta con ventajas comparativas reveladas, pero del cual se supone que lograrán dichas ventajas, el subíndice $j$ se refiere a los otros bienes no exportados con ventajas comparativas reveladas y el subíndice $i$ se refiere a todos los bienes.

\subsection{Una mirada al Espacio del Producto desde las capacidades tecnológicas}

Los autores de Harvard ligan el concepto de proximidad al enfoque neoclásico $\mathrm{y}$, en última instancia, sus fundamentos micro son las fallas de coordinación y los derrames de información (tal como plantean Hausmann y 
Rodrik (2006)). En Hausmann y Klinger (2007) se presenta un modelo neoclásico, con racionalidad sustantiva e información perfecta, para ilustrar las posibilidades de la transformación productiva y discutir con los supuestos que adoptan otros modelos neoclásicos. En ese modelo la fuente de la proximidad es la existencia de un factor de producción específico. Por otro lado, en Hidalgo et al. (2007) y en Hausmann y Klinger (2006) los autores plantean que la fuente de la proximidad está "basada en resultados" y, por lo tanto, es agnóstica respecto a las fuentes de similitud de los productos.

En el presente trabajo, se entiende que la fuente de la proximidad de los bienes proviene de las capacidades tecnológicas necesarias para su producción. Tal como se planteara, las empresas acumulan durante su trayectoria capacidades tecnológicas y las mismas son las que le permiten la producción de los bienes. Así, el parentesco de los bienes viene dado por la "similitud" entre las capacidades tecnológicas necesarias para su producción. De esta manera, la forma del Espacio del Producto, en la cual existen algunas partes densas y otras partes dispersas, implica que algunas capacidades tecnológicas permitan la elaboración de un conjunto más amplio de bienes que otras.

En esta relectura, el PRODY continúa siendo un indicador de cuán sofisticado es un bien en términos de la productividad media en su producción en el mundo. Que un bien presente un PRODY elevado significa que ese bien es producido por países con elevado producto per cápita. Por su parte el EXPY refleja el nivel de sofisticación de esta canasta de bienes. Dado que, es un promedio ponderado construido a partir del PRODY de los bienes exportados, se aproxima al valor promedio de la productividad de la canasta de exportaciones.

La lejanía refleja el grado de similitud que existe entre las capacidades tecnológicas ya acumuladas por un país y las necesarias para la producción de un bien. Por su parte, el open forest señala el valor de la proyección del cambio estructural, a partir de la distancia y la sofisticación de los bienes que no se producen respecto a los producidos, funcionando entonces como un indicador de las capacidades tecnológicas actuales de una economía. Por último, el valor estratégico de un bien se interpreta como el valor de las capacidades tecnológicas asociadas a la producción potencial de ese bien, medido a partir de la sofisticación de los bienes que estas capacidades contribuyen a desarrollar.

\subsection{Decisiones metodológicas y limitaciones del marco analítico propuesto}

Los datos de las exportaciones que se utilizan aquí son los mismos que usan Hidalgo et al. (2007) hasta el año 2000, es decir, los que se obtienen de las 
tablas de los flujos del comercio mundial de Feenstra et al. (2005), que se encuentran disponibles a nivel del producto a 4 dígitos de la clasificación CUCI revisión 2. Para el período 2000-2006 se utilizan datos asimilables extraídos de COMTRADE. De esta forma, la primera limitación encontrada proviene del relativamente alto nivel de desagregación de los datos.

Se encuentran también limitaciones vinculadas al año de referencia para la construcción de los indicadores. En el presente trabajo, se utiliza el año 2000 para la construcción del PRODY y el período 1998-2000 para la construcción de las proximidades, siguiendo a Hidalgo et al. (2007). Esta decisión puede limitar el carácter dinámico del análisis, ya que la evaluación de los $P R O D Y$ de forma más frecuente podría permitir un mejor análisis de la evolución de las capacidades tecnológicas.

Por otro lado, existen problemas vinculados a la elección de las exportaciones para aproximarse a la estructura productiva. Estos datos dejan de lado dos aspectos fundamentales para el estudio del cambio estructural, el mercado interno y los servicios, por lo que el análisis propuesto estará lógicamente incompleto. Adicionalmente, es importante aclarar que los datos de las exportaciones, incluso considerando las ventajas comparativas reveladas, pueden no representar los efectos de los aprendizajes tecnológicos, sino los mecanismos de protección y subsidio de los países a sus producciones. No obstante lo anterior, los datos de exportaciones se consideran mejores que otros disponibles para realizar este tipo de estudios, por ejemplo porque comparados con datos de la industria permiten tener información sobre actividades primarias, que pueden ser relevantes para países con ventajas basadas en recursos naturales, lo que termina resultando esencial para la aplicación empírica de este trabajo.

Por último, es importante realizar una aclaración. Con el fin de abreviar el lenguaje y exponer los resultados de forma más clara, este trabajo tratará de "bienes producidos" por un país en un momento del tiempo a aquellos "bienes que son exportados con ventajas comparativas reveladas" (con RCA $>1$ ), por ese país en ese momento. De esta manera los bienes considerados como producidos, serán denotados con el criterio de $x_{c, i, t}=1$. Esto debe tenerse en cuenta al interpretar las conclusiones del trabajo.

\section{Selección del grupo de países con especialización productiva similar a Uruguay}

En primer lugar, es necesario distinguir cuáles son los países que poseen una especialización similar a la de Uruguay. Para esta tarea se utiliza el Modelo 
Ricardiano con Heterogeneidad Estructural (MRHE), utilizado en numerosos trabajos recientes de la CEPAL para ilustrar el proceso de cambio estructural. Este modelo posibilita una caracterización de países respecto al tipo de especialización que poseen.

El modelo parte de suponer una economía internacional conformada por dos países, el Norte (centro) y el Sur (la periferia). Estas economías producen un gran número de bienes, utilizando un único factor de producción (trabajo). Los requerimientos unitarios de trabajo para la producción de cada bien se denominan $a$ en el caso del Sur y $a^{*}$ en el caso del Norte. La variable $A$ se define como el cociente entre los requerimientos del Norte respecto a los del Sur para cada tipo de bien, o lo que es lo mismo, el cociente de la productividad del país del Sur dividido por la productividad del país del Norte. De esta forma cuanto más elevada sea la curva $A$ mayor será la ventaja comparativa del Sur respecto al Norte en la producción de un bien en particular (Ecuación 1).

Para que el dominio del eje de las abscisas se encuentre entre cero y uno, todos los bienes existentes en el modelo $(n)$ se numeran de acuerdo a un subíndice $z$, el cual se encuentra normalizado para que asuma valores entre cero y uno. De esta forma, los $n$ bienes se ordenan recibiendo un valor del subíndice $z$ entre cero y uno, desde los bienes en los cuales el Sur presenta la mayor productividad relativa hacia los bienes en los cuales el Sur presenta la menor productividad relativa.

$$
\text { (Ecuación 1) } \quad A=\left(\frac{a_{z}^{*}}{a_{z}}\right)
$$

Un supuesto del modelo es que la curva $A$ y, por lo tanto, las productividades del trabajo relativas, dependen de la brecha tecnológica $(G)$ entre el Norte y el Sur (Ecuación 2). De esta forma, cuando el Sur logra una reducción de la brecha tecnológica, se produce un corrimiento de la curva $A$ para todos los bienes, o lo que es lo mismo, mejora la ventaja comparativa del Sur en la producción de todos los bienes.

$$
\text { (Ecuación 2) } \quad A=f(G)
$$

De acuerdo a la ordenación planteada en el eje de las abscisas, la representación de la curva $A$ en el dominio $z$ es una curva descendente y la pendiente de dicha curva expresa la tasa con la cual el Sur pierde ventaja comparativa con respecto al Norte a medida que se avanza hacia sectores más intensivos en tecnología. 
Dentro de este modelo, la especialización de un país se determina al saber hasta qué bien z produce. Sin embargo, para esto además de las productividades relativas es necesario considerar los salarios relativos, ya que los mismos definen los costos unitarios de la producción. La variable $W$ representa el salario relativo del Sur respecto al Norte y se construye a través del cociente entre los salarios en el Sur y los del Norte. La curva $W$ aumenta monótonamente con $z$ porque la demanda de trabajo aumenta al realizarse la diversificación productiva de una economía del Sur. Así, el incremento de la cantidad de bienes, que el Sur realiza de la producción mundial, es acompañado por un incremento de los salarios relativos respecto al Norte ${ }^{1}$.

$$
\text { (Ecuación 3) } W=\left(\frac{w_{z}}{w_{z}^{*}}\right)
$$

El modelo supone que no existe crecimiento poblacional (modelo estático), en que la economía internacional funcione en competencia perfecta (lo cual asegura el pleno empleo), los precios y los costos unitarios de producción se igualan y los bienes son producidos en la economía en la cual su costo unitario de producción es menor. De esta forma, el costo unitario de producción de un bien determinado en el Sur (por ejemplo el bien $z$ ) es igual a los requerimientos técnicos, o sea el número de horas necesarias para producir una unidad de ese bien en el Sur $\left(a_{z}\right)$, multiplicado por el salario por hora del Sur $(w)$. Así, el costo de producción de ese bien $z$ en el Sur es $w a_{z}$. Análogamente, el costo de producir ese bien $z$ en el Norte será $w a_{z}{ }^{*}$. De esta forma, un bien será producido en el Sur si $w a_{z}<w a_{z}{ }^{*}$, o lo que es lo mismo:

$$
\text { (Ecuación 4) } \frac{w}{w^{*}}<\frac{a_{z}^{*}}{a_{z}}
$$

Recordando las definiciones establecidas para $A$ y para $W$, la producción de un bien se realiza en el Sur hasta el punto en el cual $W$ iguala a $A$, lo que significa

(1) El modelo Ricardiano realiza algunas asunciones especiales para arribar a estas conclusiones, más allá de los habituales supuestos neoclásicos. En primer lugar, se asume que el ordenamiento de los bienes no es relevante ya que es posible construir una curva decreciente para todos los países. En particular, el modelo no considera las diferencias intrínsecas del bien en cuanto a su aporte a la convergencia, sino que supone que los diferentes bienes son equivalentes y que lo significativo es qué porcentaje de la variedad total de bienes produce cada país de forma eficiente con los recursos dados. En este sentido, y en segundo lugar, el modelo no incorpora el crecimiento de la población, ni la existencia de países en los cuales existe mano de obra excedente relativa, que al incorporarse al mercado de trabajo pueden provocar un aumento de la participación de estos países en la producción total de bienes, sin generar un incremento del salario real. En Holland y Porcile (2005) se reconoce esta situación en el caso de países que poseen una gran abundancia en mano de obra (Holland y Porcile, 2005, p. 44). 
que los salarios relativos del Sur (costos de producción) son menores o se igualan a las productividades relativas de ese bien en el Sur.

La Figura 2 ilustra la determinación de la especialización productiva de ambos países. En el momento inicial, el Sur produce hasta los primeros $c$ bienes, mientras que el Norte produce los bienes entre $c$ y 1 .

Figura 2

Competitividad sistémica y espuria en el Modelo Ricardiano

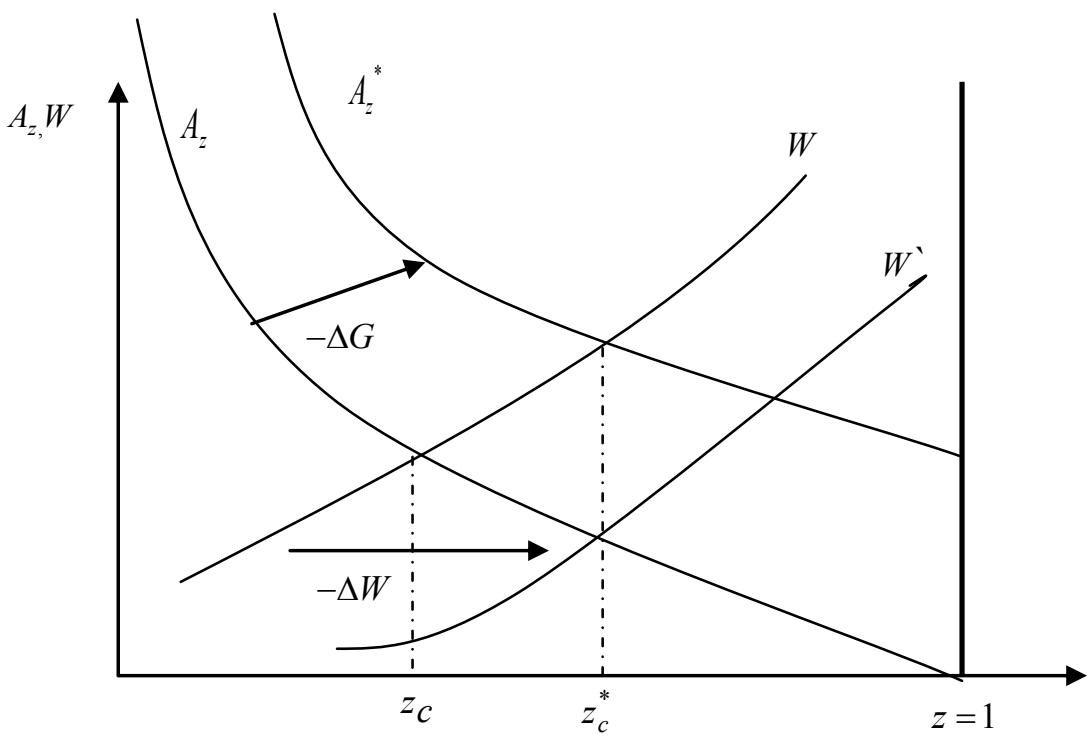

Fuente: Holland y Porcile (2005).

En Cimoli (1988) y Cimoli y Soette (1992) se estudia la inclinación de la curva $A$, la cual se denomina "multiplicador de la brecha tecnológica". Este multiplicador puede ser interpretado como la elasticidad del ratio de ventajas comparativas respecto a los bienes (ordenados de acuerdo a las ventajas comparativas del Sur). De esta forma, el multiplicador de la brecha tecnológica es una propensión al cambio del patrón de especialización frente a un cambio en los salarios relativos. Analizándola desde la perspectiva del marco teórico antes establecido, la posición e inclinación de la curva $A$ se ve afectada por las características estructurales de las economías del Sur, en donde asumiría dos características: a) ocuparía una posición muy próxima al origen, debido al carácter especializado de estas economías; b) poseería una fuerte inclinación, o sea un elevado multiplicador de la brecha tecnológica, debido a la heterogeneidad estructural que refleja la brecha tecnológica. 
Figura 3

Modelo Ricardiano con heterogeneidad estructural

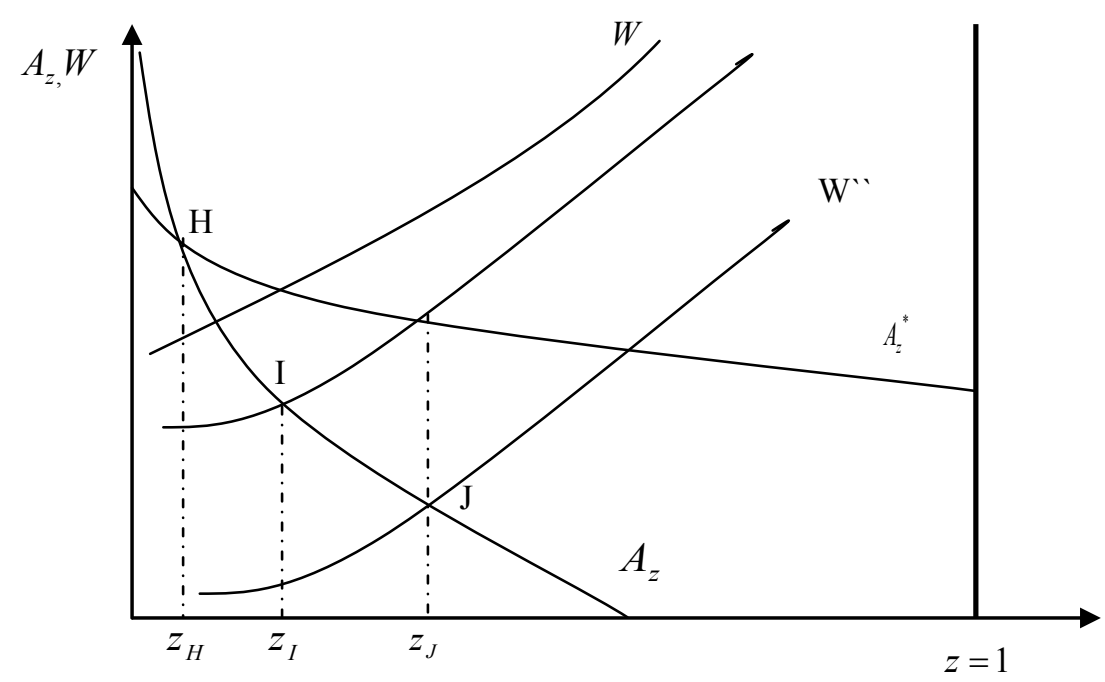

Fuente: Cimoli (2005).

En la Figura 3, la curva $A_{z}$ representa la situación de heterogeneidad y especialización estructural, debido a su cercanía al origen y su inclinación. De esta forma, cambios en el salario relativo no provocan cambios de significación en el patrón de especialización. Por otro lado, la curva $A_{z}^{*}$, que representaría la situación de homogeneidad estructural, se sitúa más a la derecha y tiene una menor pendiente.

Esta figura permite ilustrar entonces las diferencias en el cambio del patrón de especialización que derivan de la consideración de las diferencias estructurales en el modelo. Partiendo del punto H, una disminución del salario en el Sur, desde $W$ a $W$, provoca, en la economía con heterogeneidad estructural, un cambio poco significativo del patrón de especialización que pasa desde $\left(0, z_{H}\right)$ hasta $\left(0, z_{I}\right)$. En la economía con homogeneidad estructural, este mismo cambio provoca un pasaje desde $\left(0, z_{H}\right)$ hasta $\left(0, z_{J}\right)$. En este sentido, para que la economía con heterogeneidad estructural pudiera alcanzar una especialización $\left(0, z_{J}\right)$ debería estar dispuesta a aceptar un salario relativo representado por $W^{\text {" }}$ (o sea incrementar su competitividad de forma espuria).

Con respecto a la muestra inicial de países a considerar para la selección, se optó por comenzar con una muestra grande de países. Otros trabajos que buscaron establecer conjuntos de países a través de su similitud en cuanto a su especialización, como Mondelli y Rodríguez (2008) o CEPAL (2007), utilizan como muestra a los países de la OCDE, y algunos países asiáticos y de América Latina. Para el análisis aquí planteado se parte de una muestra más amplia de países, que incluye las muestras de los trabajos de Mondelli y Rodríguez (2008) y 
CEPAL (2007), y las complementa con algunos países que han sido estudiados en términos de la transformación productiva por los trabajos de los investigadores de la Universidad de Harvard. La muestra de países quedó conformada por cincuenta y tres países en los cuales se encuentran países de la OCDE, los países del Este asiático y los países de América Latina ${ }^{2}$.

Para realizar una estimación de la curva $A$, en primer lugar, es necesario calcular la productividad del trabajo, o los requerimientos unitarios del trabajo, para todos los países de la muestra. El año utilizado para realizar este ejercicio es el 2000, debido a que es el tomado como base para el cálculo de las proximidades del Espacio del Producto. Sin embargo, la no disponibilidad de datos para algunos años llevó a tomar para algunos países el año disponible más cercano al año 2000.

Tal como señala la Ecuación 5, la productividad del trabajo (PDT), se calcula como el valor agregado bruto (VAB) dividido por la cantidad de trabajadores (Empleo) para cada rama industrial de la Clasificación CIIU Rev. 2 para cada país. Los requerimientos de trabajo, a su vez, se definen como la inversa de la productividad del trabajo, o sea la cantidad de trabajadores divido al valor agregado para cada rama industrial de la CUCI Rev. $2^{3}$.

$$
\text { (Ecuación 5) } P D T_{c, i}=\frac{V A B_{c, i}}{E M P L E O_{c, i}}
$$

donde $V A B$ es el Valor Agregado Bruto, EMPLEO es la cantidad de empleados, el subíndice $i$ indica la rama industrial de la Clasificación CIIU Rev. 2 y el subíndice $c$ indica el país de la muestra.

La clasificación de CEPAL (2007), que utiliza datos de CIIU Revisión 2 a tres dígitos, se basa en Katz y Stumpo (2001). Distingue entre Industrias Intensivas en Recursos Naturales (RRNN), Industrias Intensivas en Mano de Obra (MMOO) e Industrias Difusoras del Conocimiento (CONC). La participación de los sectores CONOC en el valor agregado de la industria se utiliza en los trabajos de la CEPAL como un indicador de las capacidades tecnológicas, ya que se entiende que "la participación de los sectores CONOC en el valor agregado de la industria manufacturera mantiene una alta correlación con la capacidad de la estructura productiva para generar conocimientos, producir externalidades y competir internacionalmente sobre bases tecnológicas, y no solo a partir de la dotación original de factores. Tales son los fundamentos de la competitividad auténtica

(2) Austria, Alemania, Australia, Canadá, Chile, China, Colombia, Costa Rica, Holanda, España, Hungría, India, Irlanda, Israel, Italia, Japón, Corea, Malasia, México, Noruega, Polonia, Portugal, Singapur, España, Suecia, Turquía, Reino Unido, Estados Unidos, Uruguay, Vietnam, Belice ,Dinamarca ,Paraguay ,Nueva Zelanda ,Guatemala, Nicaragua , Argentina, Bolivia, Brasil, Ecuador, Egipto, El Salvador, Francia, Grecia, Honduras, Islandia , Panamá, Perú, Filipinas, Sudáfrica, Suiza, Venezuela.

(3) Los datos sobre la industria fueron tomados del programa INDSTAT3 2005 ISIC Rev.2 cuyo copyright pertenece a la Agencia de Naciones Unidas para el Desarrollo Industrial (ONUDI). 
(Fajnzylber, 1990), asociada a una mayor probabilidad de convergencia a largo plazo con las economías desarrolladas" (CEPAL, 2007, p. 54)

Una vez adoptadas estas decisiones, se obtiene para cada país de la muestra una estimación de la productividad del trabajo promedio para los tipos de bien RRNN, MMOO y CONOC a partir de promediar la PDT de cada rama que compone estas categorías (ver Ecuación 6).

$$
\text { (Ecuación 6) } \quad P D T_{c, k}=\sum_{n_{k}} \frac{V A B_{c, i}}{E M P L E O_{c, i}} / n_{k}
$$

donde $P D T_{k}$ es la productividad del trabajo promedio en cada uno de los sectores de la clasificación de la CEPAL (2007). El subíndice $k$ se refiere a cada una de las ramas de los sectores de la taxonomía de CEPAL (2007): RRNN, MMOO y CONOC. Por su parte, el subíndice $i$ se refiere a las ramas industriales de la clasificación CIIU Rev. 2 y el subíndice $c$ al país al que se hace referencia. Por último, $n_{k}$ se refiere a la cantidad de ramas industriales ( $i$ ) que integran los $k$ sectores.

La estimación de la curva $A$ requiere o bien la realización del ejercicio para los países uno en comparación a otro, o bien la elección de un país de referencia (Norte). Como el objetivo de este apartado es distinguir aquellos países que presentan una estructura productiva "similar" a la de Uruguay, se optó por el camino de elegir un país que se tomará como Norte del modelo para comparar con Uruguay y los otros países.

El país que se elige como referencia es Finlandia pues se entiende que constituye un ejemplo para el desarrollo de una economía como la de Uruguay, al haber llevado a cabo un proceso de crecimiento convergente basado en un cambio estructural, pasando de una economía basada en recursos naturales hacia una economía intensiva en conocimiento, sin sacrificar los rasgos básicos de su sistema de bienestar.

A continuación, se procedió a calcular las productividades relativas para las categorías RRNN, MMOO y CONOC de los demás países de la muestra con respecto a Finlandia (Ecuación 7) lo que se utiliza como estimación de la curva $A$, siguiendo la siguiente ecuación:

$$
\left(\text { Ecuación 7) } \quad A_{k}^{c, \text { Finlandia }}=\frac{P D T_{c, k}}{P D T_{\text {Finlandiak }}}\right.
$$

donde $A_{k}^{c \text {,Finlandia }}$ representa la productividad relativa del país $c$ en términos relativos a Finlandia en el sector $k$. El subíndice $k$ representa los sectores de la taxonomía de CEPAL (2007), el subíndice $c$ representa los países que se incluyen en la muestra y $P D T^{\text {Finlandia,k }}$ representa la productividad del trabajo de Finlandia para cada sector $k$. 
Como el objetivo es encontrar qué países presentan estructuras productivas "similares" a la de Uruguay, es importante analizar cómo es la curva $A$ para este país, o sea cuál es la estimación de $A_{R R N N}^{\text {Uruguaßsinlandia }}, A_{M M O O}^{\text {UruguayFinlandia }}$ y $A_{\text {CONOC }}^{\text {UruguanFinlandia }}$

Respecto a Finlandia, Uruguay presenta la mayor productividad relativa en bienes tipo RRNN $(1,7)$, la segunda mejor en bienes tipo MMOO $(0,6)$ y el peor desempeño relativo en bienes tipo $\operatorname{CONOC}(0,3)$. De esta forma, la curva A de Uruguay presenta un eje de las abscisas ordenado desde bienes tipo RRNN hacia bienes tipo CONOC, según muestra el Gráfico 1.

\section{Gráfico 1}

Productividad industrial de Uruguay respecto a Finlandia año 2000 (por tipo de bien según CEPAL, 2007)

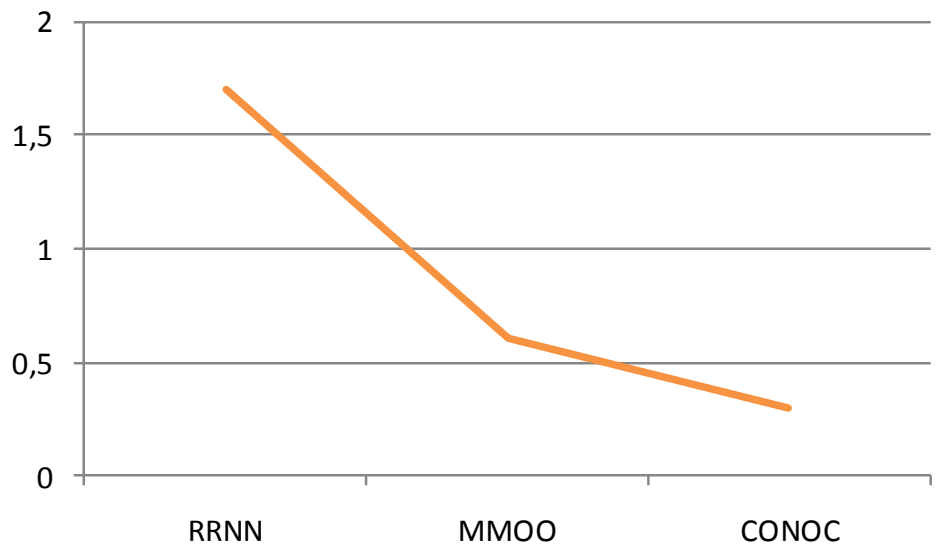

Fuente: Elaboración propia en base a ONUDI (INDSTAT3, 2005).

A partir de esta estimación, es necesario definir cuáles son las economías que presentan estructuras productivas similares a Uruguay. Para ello se toma en cuenta la dinámica del cambio estructural que predice el MRHE. El primer criterio que se adoptó fue descartar aquellos países que presentan un ordenamiento diferente al de Uruguay en el eje de las abscisas de la curva $A$, dado que, de acuerdo al modelo, el proceso de cambio estructural se realiza a través de la reducción de la brecha tecnológica y de la diversificación de la producción hacia los bienes que se ubican a la derecha. En este marco, los países que no presentan el mismo orden de la curva $A$ que Uruguay, presentan diferentes procesos de cambio estructural y, por lo tanto, se descartan como estructuras productivas similares a la uruguaya.

De esta forma, el grupo de países no descartados presenta el mismo ordenamiento en el eje de las abscisas que Uruguay. Sin embargo, para analizar si efectivamente estos países presentan una estructura productiva similar a Uruguay, es necesario evaluar la magnitud de las productividades relativas para cada tipo de bien. Esta evaluación se realiza a través de un criterio basado en las ventajas 
comparativas. Se entiende que un país presenta ventaja comparativa respecto a Finlandia en un bien cuando la productividad relativa es mayor que uno para ese tipo de bien.

Finalmente, los países seleccionados son: Argentina, Australia, Chile, Dinamarca, España, Nicaragua, Nueva Zelanda, Perú, Uruguay y Turquía. Estos países presentan ventaja absoluta en los bienes tipo RRNN y no la poseen en los otros tipos de bienes. Estos casos son interpretados como países que no tienen brecha tecnológica en el caso de la producción de los bienes tipo RRNN, pero si presentan una brecha significativa en la producción de los otros tipos de bienes. En promedio, estos países tienen una productividad del 177\% respecto a Finlandia en el caso de los bienes RRNN, pero apenas llegan al 71\% en el caso de los bienes MMOO y al 53\% en el caso de bienes tipo CONOC.

De esta forma, se interpreta que los integrantes del grupo antedicho poseen una fuerte heterogeneidad estructural, con elevada productividad en los bienes basados en Recursos Naturales y una fuerte brecha tecnológica en los bienes basados en la Mano de Obra y el Conocimiento.

\section{Descripción de los países a analizar a través del instrumental propuesto}

Realizando una comparación respecto al EXPY de estos países seleccionados (Gráfico 2), se visualiza que los países se encuentran en tres niveles diferentes: países con un alto nivel de EXPY (Dinamarca, España y Nueva Zelanda), con nivel medio (Argentina, Australia, Turquía y Uruguay), y nivel bajo (Chile, Perú y Nicaragua). Esto implica que el nivel promedio de la productividad de la canasta de exportación de los diez países presenta algunas diferencias entre sí, que en general se mantienen en el período de tiempo analizado.

Gráfico 2

Evolución del EXPY de los países con especialización similar 1986-2005

(Promedios trianuales)

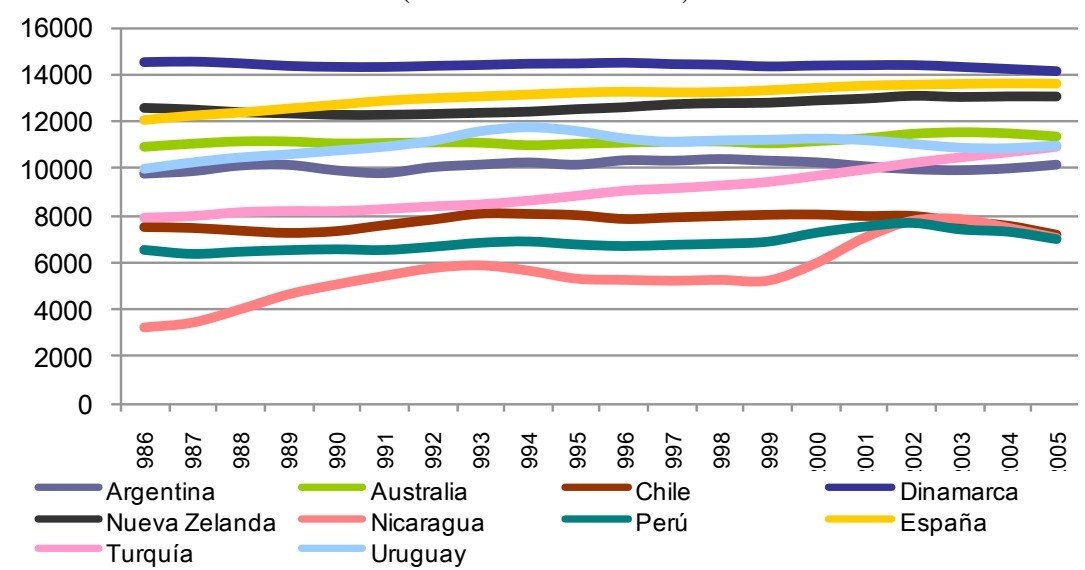

Fuente: Elaboración propia en base a Feenstra et al. (2005), Comtrade e Hidalgo et al. (2007). 
La diferencia entre los EXPY de los países no proviene mayormente de los tipos de bienes que exportan, sino de la ponderación que presentan los bienes en sus canastas de exportación. Por esto, se analiza el grado de concentración de las exportaciones por bienes, encontrándose diferencias significativas en el grado de concentración de la canasta de exportaciones de estos países ${ }^{4}$ (Cuadro 1). Los tres países que presentan una mayor concentración son Chile, Nicaragua y Perú, siendo también los mismos países que exhiben el peor desempeño en el EXPY. Aunque menor a la de estos países, Uruguay también presenta una canasta concentrada, en nivel cercano al de Perú. En el otro extremo se encuentran Dinamarca, España y Turquía. Argentina y Nueva Zelanda poseen valores similares entre ellos, con un bajo nivel de concentración. De esta forma, el grado de concentración de la canasta de exportación parece jugar un rol relevante para explicar el nivel de EXPY que alcanzan estos países.

Cuadro 1

Concentración de las canastas de exportación

\begin{tabular}{|c|c|}
\hline País & $\begin{array}{c}\text { Índice de Concentración de la canasta } \\
\text { de exportaciones }(\mathrm{H})\end{array}$ \\
\hline Argentina & 0,029 \\
\hline Australia & 0,0476 \\
\hline Chile & 0,3478 \\
\hline Dinamarca & 0,0145 \\
\hline España & 0,0229 \\
\hline Nicaragua & 0,107 \\
\hline Nueva Zelanda & 0,0269 \\
\hline Perú & 0,0834 \\
\hline Turquía & 0,0167 \\
\hline Uruguay & 0,0782 \\
\hline
\end{tabular}

Elaboración propia en base a Hidalgo et al. (2007) y Comtrade.

El ordenamiento de los países de acuerdo al nivel del open forest (Gráfico 3), que como fue dicho, mide el valor de la proyección del cambio estructural, es similar al que se encuentra cuando se analiza el EXPY, aunque no estrictamente igual. Chile, Perú y Nicaragua poseen los niveles más bajos mientras que España y Dinamarca, y en este caso también Turquía presentan valores más elevados lo que les otorga un mejor panorama en términos de la proyección de su cambio

(4) Para estimar el grado de concentración de la canasta de exportaciones su utiliza el Índice de Herfindal. $H=\sum_{i=1}^{n} p_{i}^{2} ;$ donde $p i=\frac{e_{i}}{V T} ; V T=\sum_{i=1}^{n} e_{i} ; e_{i}$ es el valor de las exportaciones del bien $\mathrm{i}$; VT es el valor total de las exportaciones del país; $H$ es el Índice de Herfindal. Además $1 / n \leq H \leq 1$. 
estructural. Se puede interpretar que el elevado valor de Turquía en este indicador es lo que explica el crecimiento de su EXPY antes comentado, ya que como se estableció, el open forest es un buen predictor del EXPY.

\section{Gráfico 3}

Evolución del open forest de los países con especialización similar 1986-2005

(Promedios trianuales)

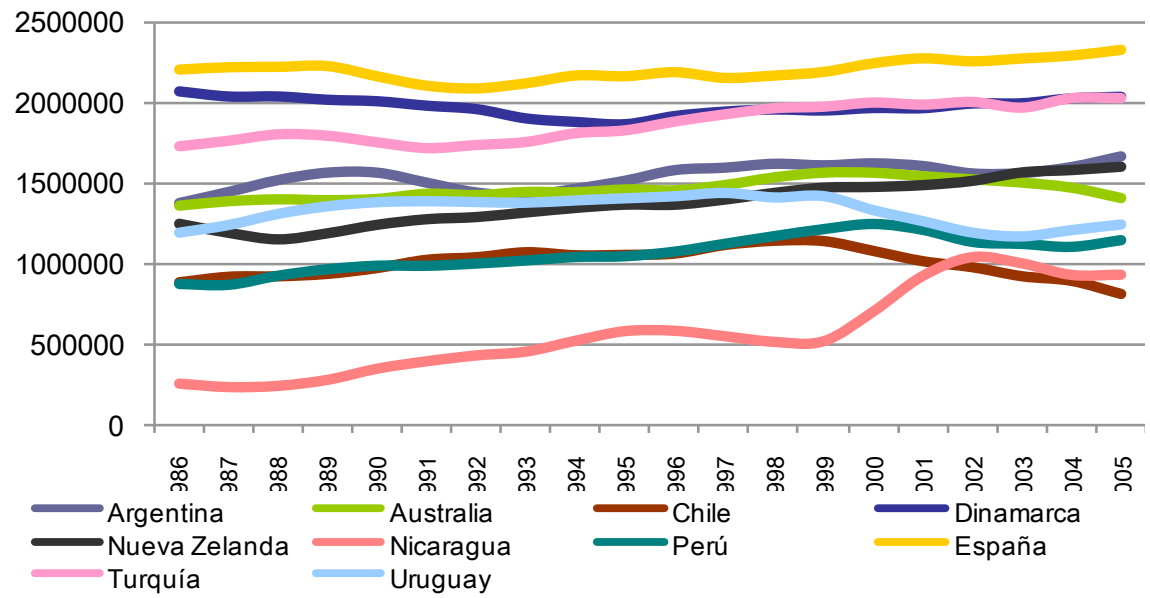

Fuente: Elaboración propia en base a Feenstra et al. (2005), Comtrade e Hidalgo et al. (2007).

\section{Potencialidades para el cambio estructural}

Al ser el open forest de cada país un buen predictor del nivel de sofisticación de la canasta de exportaciones de cada país, resulta una variable adecuada para "proyectar" el cambio estructural de los países, aunque desde una perspectiva más bien estática. Al tener como foco de análisis a las potencialidades para el cambio estructural, se evalúa necesario para imprimirle mayor carácter dinámico al análisis, permitir a los países alcanzar un conjunto determinado de bienes, denominados "bienes estratégicos", analizando posteriormente los impactos para el cambio estructural que ello implica.

\subsection{Las fronteras de eficiencia y los bienes estratégicos}

En algunos textos recientes (Hausmann y Klinger, 2006, 2007; Hausmann, 2007), que analizan las estrategias del desarrollo productivo de un país, se plantea que existe una "frontera de eficiencia" que delimita "bienes estratégicos", considerando la distancia en la que se encuentran los productos y lo valiosos que éstos son.

En primer lugar, analizan cuáles son los bienes que el país no exporta con ventajas comparativas reveladas y que se encuentran cercanos a la actual canasta de exportaciones del país. Los autores consideran bienes cercanos a aquellos que 
cumplen la condición de presentar una lejanía inferior a la media menos $n$ desvíos estándar de la variable (en sus trabajos se han utilizado valores de 1, 1,5 y 2 para $n$, en este estudio se utilizó un valor de $0.5^{5}$ ).

Sin embargo, estos bienes no se constituyen en bienes estratégicos solamente por estar cerca de la canasta de exportaciones sino que es necesario que sean valiosos en términos del cambio estructural. Se consideran valiosos aquellos bienes que presentan un nivel de PRODY mayor al EXPY de ese país y, de esa manera, su producción podría contribuir a incrementar el nivel de sofisticación de la canasta de exportaciones de ese país, o sea, contribuir para reducir su brecha tecnológica con los países más avanzados.

En el presente trabajo se formaliza el criterio para delimitar los bienes estratégicos, a través de tres condiciones: a) que no sean exportados con ventajas comparativas por el país, b) que presenten una lejanía menor que la media menos $n$ desvíos estándar (siendo en este caso $n=0,5$ ), y c) que posean un $P R O D Y$ mayor al valor del EXPY del país en el año que se considera.

Las fronteras de eficiencia de un país se pueden representar para cada año, identificando cada bien no producido con ventajas comparativas reveladas por el país, mediante un punto en el plano establecido por un eje horizontal en el que se representa la lejanía del bien, y un eje vertical en que se representa el PRODY de los bienes (Figura 4).

Figura 4

Frontera de eficiencia tipo

Frontera

Valor estratégico vs. distancia

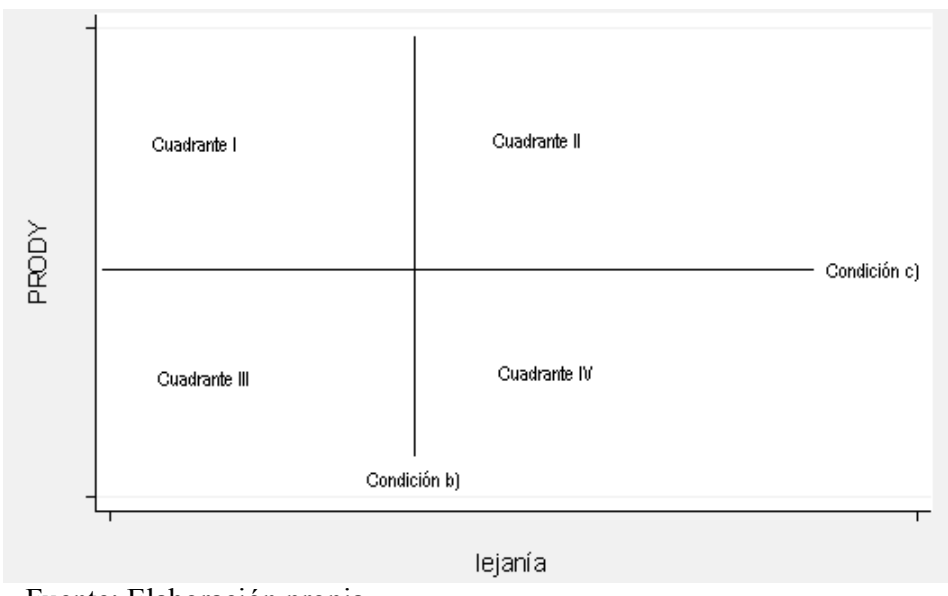

Fuente: Elaboración propia

(5) La decisión de utilizar un parámetro $n$ de 0,5 se realizó simplemente a los efectos de no exigir un valor, que excluya o incorpore una cantidad muy grande de bienes para los países. Más adelante en este capítulo, se analizará la sensibilidad de los resultados a este criterio. 
Para el análisis gráfico de las fronteras de eficiencia lo más importante a observar es la forma de la nube de puntos (que representan los bienes no producidos) y la distancia relativa de la misma al eje de las ordenadas. Cuanto menor sea la lejanía de la nube de puntos en su conjunto (cuanto más cerca se encuentre del eje de las ordenadas en el cero), más facilidades tendrá el país para alcanzar los bienes no producidos. De esta forma un país con mayores acumulaciones de capacidades tecnológicas, deberá poseer una recta de condición b) más cercana al origen, denotando que tanto los bienes valiosos como los no tan valiosos están cercanos a las capacidades ya acumuladas de ese país.

Del mismo modo, un país que presente menores acumulaciones tecnológicas presentará sus cuadrantes I y III ocupando un área mayor. Además, este tipo de países contará probablemente con la mayor parte de su nube de bienes no producidos situada en los cuadrantes II y III, lo que puede leerse como que los bienes valiosos para el país se encuentran relativamente lejos. Es esperable entonces que exista una relación positiva entre la lejanía media de la nube de puntos y su dispersión.

Los bienes estratégicos serán aquellos que pertenezcan al Cuadrante I, en términos de esta figura. Dado que la condición $b$ ) está expresada en términos relativos a la nube de puntos, este criterio no puede nunca dejar todos los bienes "de un lado de la línea". No se puede decir lo mismo de la condición c), ya que teóricamente puede ocurrir que todos los bienes de la frontera de un país presenten un PRODY menor al EXPY del país. Si bien lo anterior es altamente improbable, ayuda a explicar porqué las diferencias entre países respecto a las cantidades de bienes estratégicos que enfrentan, dependen más fuertemente de la condición $c$ ) que de la condición $b$ ). La disposición de la nube de puntos es independiente del $E X P Y$, por lo que para una misma nube, el valor del EXPY determinará fuertemente la cantidad de bienes que se considerarán estratégicos.

\subsection{Las fronteras de eficiencia para los países seleccionados}

El Gráfico 4 muestra las fronteras de eficiencia en el año 2006 para los países que se toman en este trabajo como aquellos que tienen estructuras productivas similares a Uruguay. 
Espacio del producto y cambio estructural: un enfoque latinoamericano ...

\section{Gráfico 4}

Fronteras de eficiencia para 2006

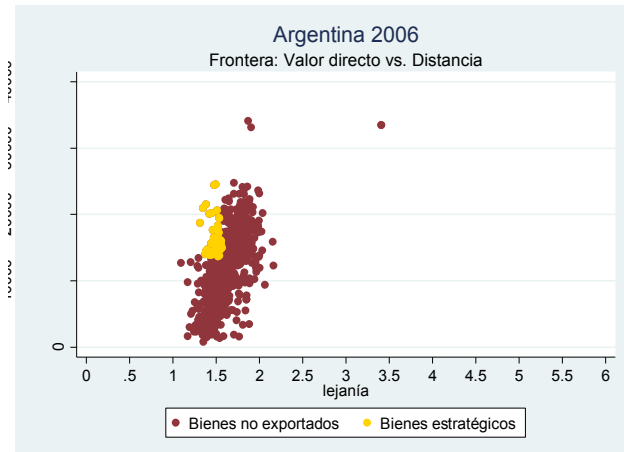

Chile 2006

Frontera: Valor directo vs. Distancia

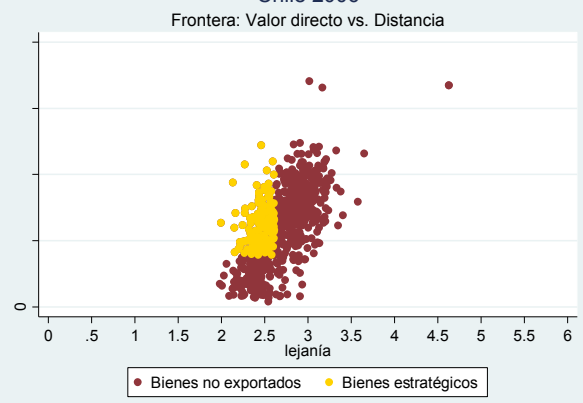

España 2006

Frontera: Valor directo vs. Distancia

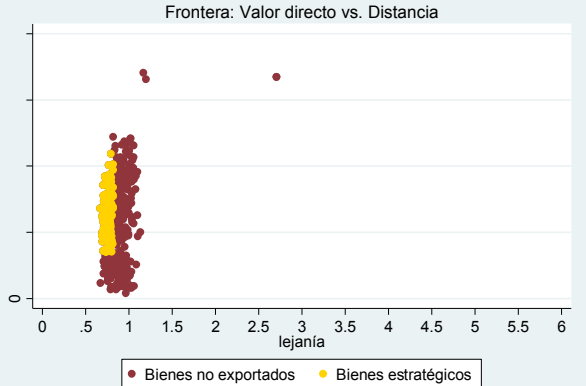

Nueva Zelanda 2006

Frontera: Valor directo vs. Distancia

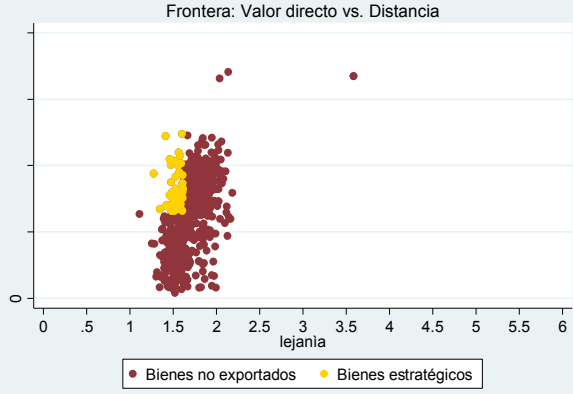

Australia 2006

Frontera: Valor directo vs. Distancia

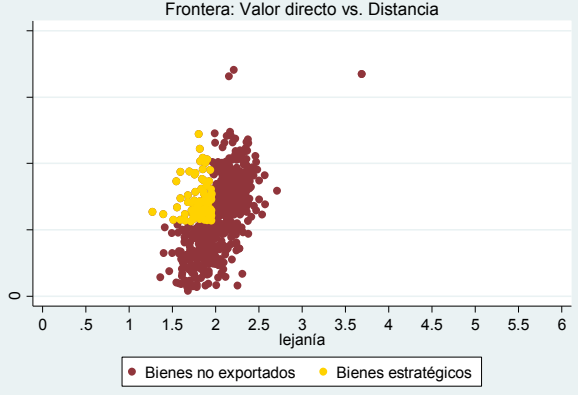

Dinamarca 2006

Frontera: Valor directo vs. Distancia

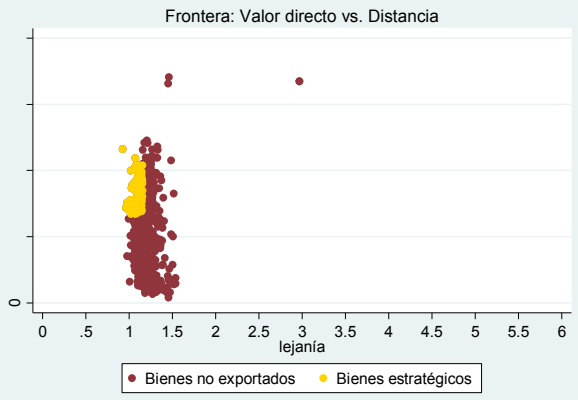

Nicaragua 2006

Frontera: Valor directo vs. Distancia

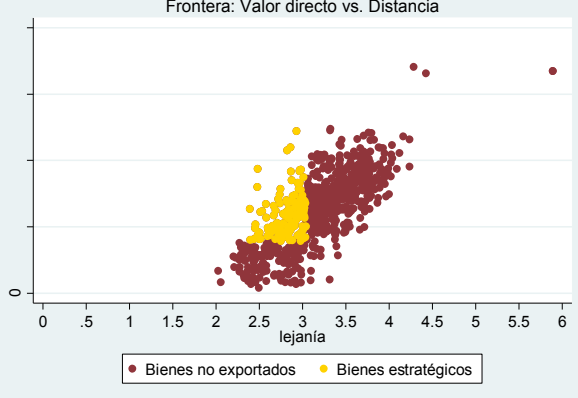

Perú 2006

Frontera: Valor directo vs. Distancia

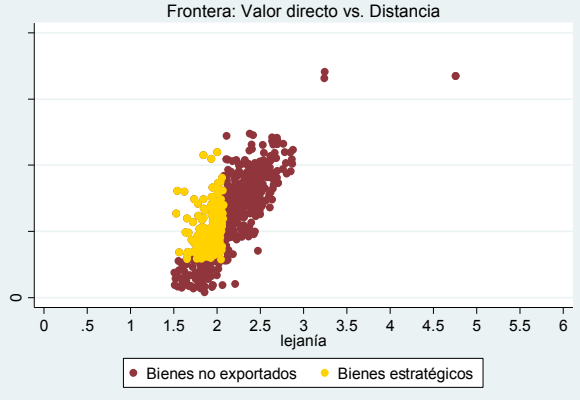



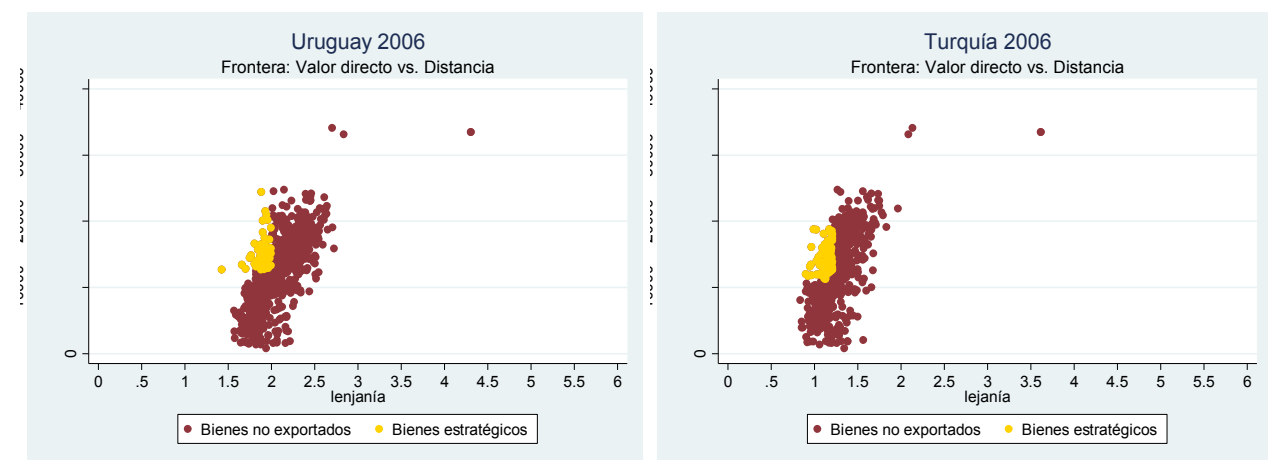

Fuente: Elaboración propia en base a Hidalgo et al. (2007) y Comtrade.

Las nubes de puntos de España, Dinamarca y Turquía se encuentran más cercanas al eje de las ordenadas que las nubes de los otros países. Contrariamente, las nubes de Chile, y fundamentalmente Nicaragua, se posicionan relativamente a una mayor distancia promedio de las capacidades tecnológicas ya acumuladas. Argentina, Australia, Nueva Zelanda y Uruguay aparecen como muy similares, con una lejanía promedio relativamente menor para el caso de Argentina.

Por otro lado, mientras que las nubes de Dinamarca y España presentan una forma compacta, nuevamente las nubes de Chile y Nicaragua dan señales menos promisorias para el cambio estructural, ya que los bienes de mayor valor se encuentran más lejos de sus capacidades acumuladas, lo que puede apreciarse en que sus nubes aparecen con una forma alargada y sugiriendo una pendiente positiva (ocupando en mayor medida los cuadrantes II y III). Argentina, Nueva Zelanda, Australia, Uruguay y en menor medida Perú, representan casos más intermedios y muestran formas muy similares en sus nubes de puntos. Esta evidencia parece reforzar la hipótesis de la existencia de una relación positiva entre lejanía media y dispersión de la nube.

Adicionalmente, los gráficos parecen reafirmar la idea de que es el nivel de EXPY del país el determinante del número de bienes estratégicos que dicho país enfrenta. Para nubes de bienes similares (como pueden resultar las de Nueva Zelanda y Argentina), los países que tienen un EXPY más bajo para su canasta de exportación (Argentina), poseen una mayor cantidad de bienes estratégicos. Así, si un país logra, con un nivel de sofisticación de su producción menor que otro, acumular aprendizajes tecnológicos que lo ubican en una situación similar en cuanto a bienes alcanzables, entonces ese país presentará mayores potencialidades para el cambio estructural porque le será más fácil producir bienes con un nivel de sofisticación de su producción. 
Las conclusiones extraídas del análisis gráfico se obtienen también cuando se realiza un análisis a través de los indicadores de posición y dispersión de la nube.

El Cuadro 2 muestra para los diez países el valor de las variables señaladas. Dado que las estructuras productivas pueden ser consideradas como similares, el conjunto de los bienes que no producen con ventajas comparativas son compartidos en general por los países, por lo que la media y el desvío estándar del PRODY no presentan diferencias marcadas.

Cuadro 2

Un set de indicadores de las Fronteras de eficiencia para el año 2006

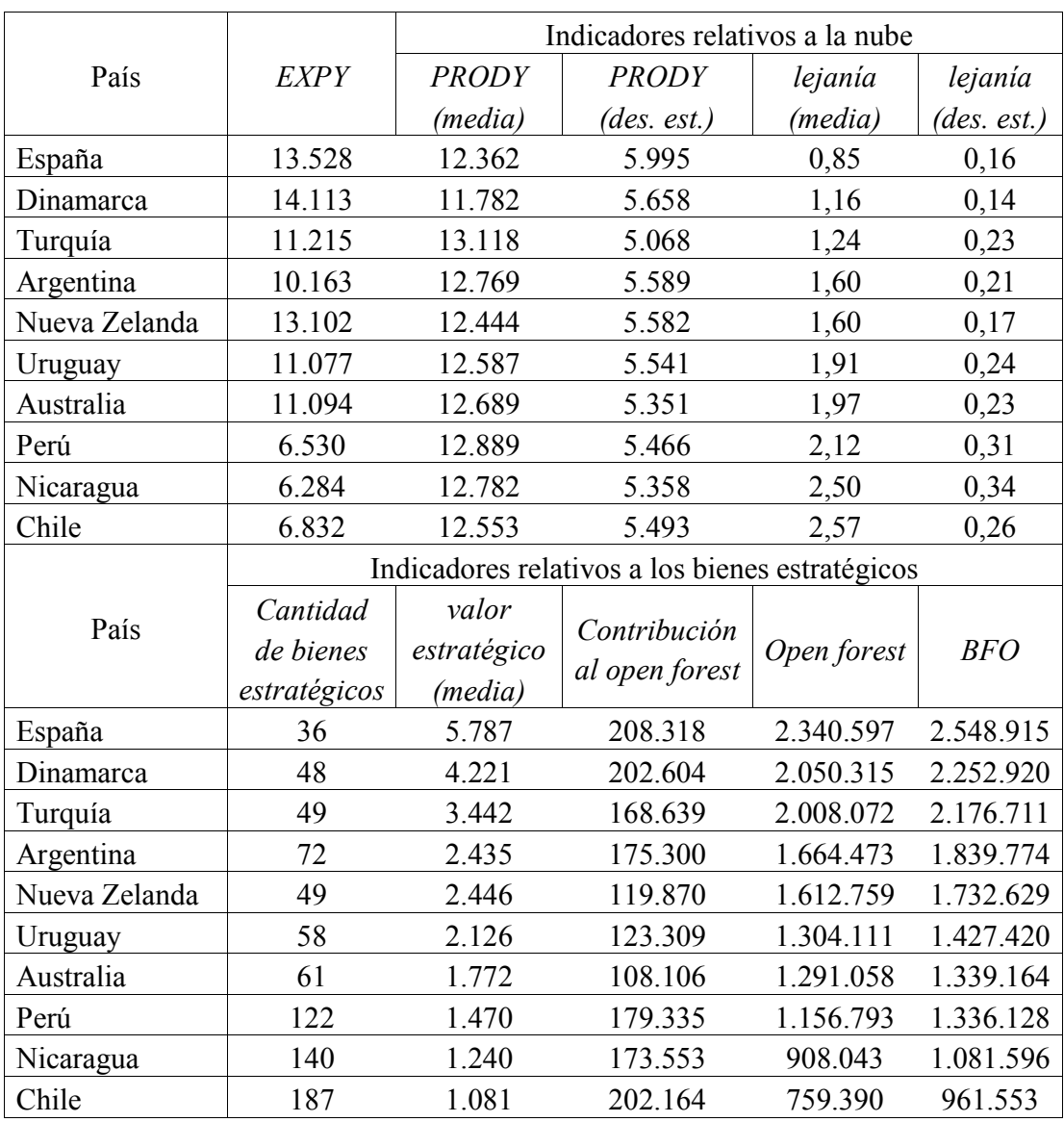

Fuente: Elaboración propia en base a Hidalgo et al. (2007) y Comtrade.

Contrariamente, la lejanía relativa que estos bienes tienen de las acumulaciones actuales de cada economía es la que determina las diferencias en las fronteras antes expuestas. Analizando los indicadores referentes a la lejanía se 
pueden reafirmar los comentarios que surgían del análisis gráfico. Mientras que España, Dinamarca y Turquía presentan valores relativamente bajos de lejanía promedio (que rondan la unidad), en el caso de Nicaragua, Chile y Perú este valor es superior a dos.

El desvío estándar de esta variable marca cuán dispersa es la nube en esta dimensión. En general, los resultados reafirman que cuanto menor es la lejanía, menores son los desvíos estándar (el coeficiente de correlación entre estas variables es de 0,83 ), lo que indica que los países que tienen los bienes no producidos más cercanos a sus capacidades tecnológicas acumuladas, adicionalmente presentan una menor dispersión entre estos bienes.

\subsection{Las potencialidades para el cambio estructural}

Para analizar las potencialidades para el cambio estructural es necesario aproximarse a cómo el desarrollo de ventajas comparativas reveladas en los bienes estratégicos favorecería el cambio estructural, lo que puede aproximarse a través del valor estratégico de esos bienes. Para sintetizar las potencialidades de una economía para el cambio estructural en un único indicador, se propone el indicador $B F O$, el cual agrega al open forest actual del país, la contribución al open forest de sus bienes estratégicos, definida como la suma del valor estratégico de los bienes estratégicos. La formalización del estadístico, para un país $c$ con $m$ bienes estratégicos, en un año $t$ es:

(9) $B F O_{c, t}=$ open_forest $_{c, t}+$ contribución_al_open_forest $t_{c, t}$

(10) contribución_al_open_forest ${ }_{c, t}=\sum_{m} v_{\text {valor_estatégico }} c, t, m$

El open forest señala el valor de la proyección del cambio estructural, a partir de la distancia y el valor de los bienes que no se producen, valorizando de esta forma las capacidades tecnológicas actuales de una economía. Por su parte, la contribución al open forest mide la suma del valor de las capacidades tecnológicas asociadas a los bienes estratégicos, medidos a partir del valor de los bienes que se pueden desarrollar en caso de comenzar a producirse los bienes estratégicos. De esta forma, el estadístico $B F O$ definido como la suma de ambos indicadores, y que mide las potencialidades para el cambio estructural, es una aproximación al valor del open forest en el caso de que se produzcan con ventajas comparativas los bienes estratégicos.

El Cuadro 2 señala cuál es el promedio del valor estratégico que aportan los bienes estratégicos para cada país. Este valor presenta una elevada correlación con los open forest de estos países $(0,94)$. Así, los países que tienen un valor de open forest relativamente más elevado, presentan bienes estratégicos con un mayor valor estratégico. 
La contribución al open forest sintetiza la información que brinda la cantidad de bienes estratégicos con el valor promedio de cada uno de ellos. Del Cuadro 2 se desprende que, permitiendo a los países desarrollar ventajas comparativas en los bienes estratégicos, no necesariamente los países que más se benefician son aquellos que tienen un open forest más elevado ni tampoco los que tienen más cantidad de bienes estratégicos (las correlaciones entre la contribución al open forest y estas variables son respectivamente de 0,22 y 0,31). De esta forma, la contribución al open forest de cada bien aporta información relevante para entender las potencialidades de estos países para el cambio estructural ${ }^{6}$.

El indicador $B F O$ para el año 2006, permite ordenar a los países de acuerdo a las potencialidades que presentan para el cambio estructural. Así, este indicador tendría que confirmar las conclusiones que se han ido extrayendo a lo largo de este capítulo. Como puede verse, España, Turquía y Dinamarca presentan mayores niveles de $B F O$ y ya presentaban los mayores niveles de open forest. En el caso contrario, Chile y Nicaragua, presentan los menores niveles de open forest, y pese a que reciben una contribución al open forest elevada, no logran revertir el panorama de escasas oportunidades para realizar el cambio estructural.

Una situación menos clara se da entre Argentina, Australia, Nueva Zelanda, Perú y Uruguay. En el caso de Argentina y Nueva Zelanda, el indicador $B F O$ incrementa las diferencias que entre estos países se perciben analizando sólo el open forest. Contrariamente, en el caso de Uruguay, Australia y Perú el indicador $B F O$ señala mayores similitudes entre ellos que los open forest.

\section{Contrastando las potencialidades para el cambio estructural}

\subsection{Pruebas de hipótesis}

En este apartado se da una respuesta definitiva a la pregunta de investigación al contrastar las potencialidades para el cambio estructural de los

(6) El criterio de que el indicador suma de las contribuciones al open forest adicione información, fue utilizado como forma de corroborar la elección hecha del parámetro $n$ que multiplica al desvío estándar de la densidad en la definición del criterio para considerar a los bienes como estratégicos. Realizado el ejercicio antes presentado para un valor $n$ de 0,3 , la suma de las contribuciones al open forest presentaba una elevada correlación con el valor de open forest promedio de los bienes $(0,81)$ y una muy menor con la cantidad de bienes $(0,11)$. A su vez, utilizando un valor del parámetro $x$ de 1 (que volvía más exigente el criterio), la suma de las contribuciones al open forest se correlacionaba fuertemente con la cantidad de bienes $(0,95)$. Más aún, en este caso el valor promedio de open forest de los bienes presentaba una correlación negativa $(-0,65)$, lo que se puede interpretar en el sentido que el indicador reproduce la información que aporta la cantidad de bienes y por lo tanto penaliza la contribución promedio. Se concluye entonces que utilizando parámetros $x$ con valores más o menos elevados que 0,5, el indicador contribuciones al open forest pierde parte de su potencia, que consiste en combinar la información que brinda la cantidad de bienes con la de la contribución promedio de cada uno de ellos. 
países analizados utilizando dos metodologías estadísticas diferentes y con el indicador $B F O$ como aproximación a dichas potencialidades. Para esto, la prueba a realizar debe identificar si la medida obtenida del indicador $B F O$ es significativamente diferente entre algunos de los países. Para un par de países $c$ y $p$, en un año $t$, se puede plantear la hipótesis nula de la prueba como:

$$
\left.H_{o}\right) B F O_{c, t}=B F O_{p, t} \quad \text { siendo } c \neq p \text {. }
$$

Si se rechaza la hipótesis nula para algún par de países, entonces debe rechazarse la hipótesis de que el conjunto tiene las mismas potencialidades para el cambio estructural. Por otro lado, la prueba tiene la posibilidad de señalar cuáles son los países del conjunto analizado, para los que no es posible rechazar que posean efectivamente las mismas potencialidades para el cambio estructural que el resto.

Las modificaciones que pueda sufrir una estructura productiva, dada la naturaleza del fenómeno, sólo se pueden considerar en períodos de tiempo relativamente largos, ya que las estructuras productivas de los países no suelen sufrir alteraciones importantes de un año a otro. Una debilidad del open forest (y por construcción, también del $B F O$ ) es que al considerar datos de exportaciones para cada año presenta demasiada volatilidad para ser un indicador de una característica estructural y, por lo tanto, relativamente permanente a largo plazo en una economía. Es por esta razón que se considera más apropiado trabajar con una medida de síntesis de $B F O$ para varios años, de modo a evitar valores del indicador afectados por coyunturas particulares. En este análisis se evalúa como suficiente trabajar con una década (desde 1997 hasta 2006) como horizonte temporal.

De esta forma, se considera el $B F O$ de cada año como una observación del estadístico $B F O$, inobservable, que refleja la potencialidad para el cambio estructural de una economía. Se supone que la verdadera potencialidad para el cambio estructural, el estadístico $B F O$, tiene una distribución normal (no se encuentran motivos para suponer una distribución distinta) y se procede a estimar su media y su desvío estándar con las observaciones del $B F O$ para cada año.

El Gráfico 5 muestra la media estimada del estadístico BFO utilizando el período 1997-2006, para los 10 países de referencia. El ordenamiento que surge del análisis de este indicador es similar al presentado en el capítulo anterior, en el cual, el período considerado era de tan sólo un año. Por basarse en información de una década, se entiende que este ordenamiento refleja mejor las potencialidades estructurales de las economías. 
Gráfico 5

Potencialidades para el cambio estructural 1997-2006

(BFO promedio del periodo)

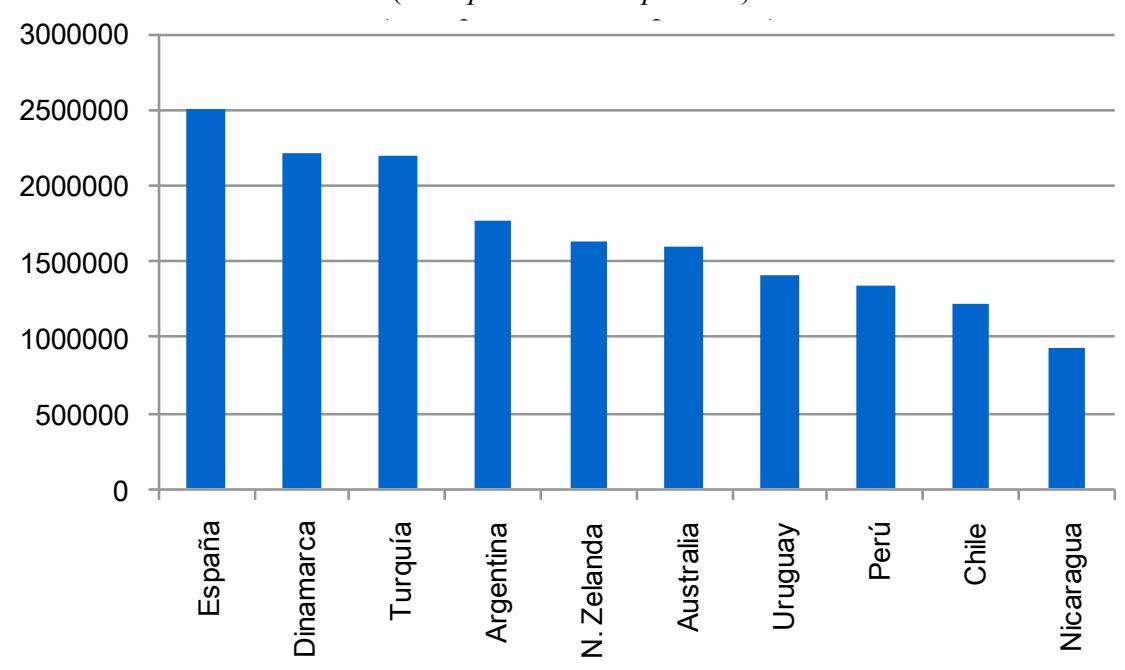

Fuente: Elaboración propia en base a Hidalgo et al. (2007) y Comtrade.

Una vez supuesta la normalidad del indicador $B F O$, se realizaron dos pruebas diferentes para contrastar las hipótesis nulas comparando medias poblacionales a través de medias estimadas. Por un lado se realizó el Contraste de Igualdad de Medias en Poblaciones Normales con Varianzas Conocidas, que ayuda a evaluar vis a vis las medias de dos países. Por otro lado, se utilizó también un test de comparación múltiple de medias denominado Contraste de Tukey, que se basa en el estadístico de rango estudentizado para el cual Tukey calcula la distribución. La diferencia principal respecto al contraste anterior consiste en que la varianza que se toma como referencia se toma de toda la muestra de países, por lo que su valor será mayor y por esto la prueba resulta más exigente. El resumen de los resultados de la primera prueba se expone en la Tabla 1 y los de la segunda en la Tabla 2.

Tabla 1

Resultados de contraste de igualdad de medias en poblaciones normales con varianzas conocidas

\begin{tabular}{|c|c|c|c|c|c|c|c|c|c|c|}
\hline País & $\begin{array}{c}\text { Argen- } \\
\text { tina }\end{array}$ & Australia & Chile & Dinamarca & España & Nicaragua & $\begin{array}{c}\text { Nueva } \\
\text { Zelanda }\end{array}$ & Perú & Turquía & Uruguay \\
\hline Argentina & & $\mathrm{R}$ & $\mathrm{R}$ & $\mathrm{R}$ & $\mathrm{R}$ & $\mathrm{R}$ & $\mathrm{R}$ & $\mathrm{R}$ & $\mathrm{R}$ & $\mathrm{R}$ \\
\hline Australia & $\mathrm{R}$ & & $\mathrm{R}$ & $\mathrm{R}$ & $\mathrm{R}$ & $\mathrm{R}$ & No R & $\mathrm{R}$ & $\mathrm{R}$ & $\mathrm{R}$ \\
\hline Chile & $\mathrm{R}$ & $\mathrm{R}$ & & $\mathrm{R}$ & $\mathrm{R}$ & $\mathrm{R}$ & $\mathrm{R}$ & $\mathrm{R}$ & $\mathrm{R}$ & $\mathrm{R}$ \\
\hline Dinamarca & $\mathrm{R}$ & $\mathrm{R}$ & $\mathrm{R}$ & & $\mathrm{R}$ & $\mathrm{R}$ & $\mathrm{R}$ & $\mathrm{R}$ & No R & $\mathrm{R}$ \\
\hline España & $\mathrm{R}$ & $\mathrm{R}$ & $\mathrm{R}$ & $\mathrm{R}$ & & $\mathrm{R}$ & $\mathrm{R}$ & $\mathrm{R}$ & $\mathrm{R}$ & $\mathrm{R}$ \\
\hline
\end{tabular}


Tabla 1 - Continuación

\begin{tabular}{lcccccccccc}
\hline \multicolumn{1}{c}{ País } & $\begin{array}{c}\text { Argen- } \\
\text { tina }\end{array}$ & Australia & Chile & Dinamarca & España & Nicaragua & $\begin{array}{c}\text { Nueva } \\
\text { Zelanda }\end{array}$ & Perú & Turquía & Uruguay \\
\hline $\begin{array}{l}\text { Nicaragua } \\
\text { Nueva }\end{array}$ & $\mathrm{R}$ & $\mathrm{R}$ & $\mathrm{R}$ & $\mathrm{R}$ & $\mathrm{R}$ & & $\mathrm{R}$ & $\mathrm{R}$ & $\mathrm{R}$ & $\mathrm{R}$ \\
$\begin{array}{l}\text { Zelanda } \\
\text { Perú }\end{array}$ & $\mathrm{R}$ & $\mathrm{No} \mathrm{R}$ & $\mathrm{R}$ & $\mathrm{R}$ & $\mathrm{R}$ & $\mathrm{R}$ & & $\mathrm{R}$ & $\mathrm{R}$ & $\mathrm{R}$ \\
Turquía & $\mathrm{R}$ & $\mathrm{R}$ & $\mathrm{R}$ & $\mathrm{R}$ & $\mathrm{R}$ & $\mathrm{R}$ & $\mathrm{R}$ & & $\mathrm{R}$ & No R \\
Uruguay & $\mathrm{R}$ & $\mathrm{R}$ & $\mathrm{R}$ & $\mathrm{R}$ & $\mathrm{R}$ & $\mathrm{R}$ & $\mathrm{R}$ & $\mathrm{No} \mathrm{R}$ & $\mathrm{R}$ & $\mathrm{R}$ \\
\hline
\end{tabular}

Nota: $\mathrm{R}$ significa que se rechaza la hipótesis nula para el contraste entre esos países, mientras que NO $\mathrm{R}$ significa no rechazarla. La columna sombreada es En celeste se presentan las pruebas para el caso de Uruguay.

Fuente: Elaboración propia en base a Hidalgo et al. (2007) y Comtrade.

Tabla 2

Resultados de contraste de Tukey

\begin{tabular}{|c|c|c|c|c|c|c|c|c|c|c|}
\hline País & $\begin{array}{c}\text { Argen- } \\
\text { tina }\end{array}$ & Australia & Chile & Dinamarca & España & Nicaragua & $\begin{array}{l}\text { Nueva } \\
\text { Zelanda }\end{array}$ & Perú & Turquía & Uruguay \\
\hline Argentina & & No $R$ & $\mathrm{R}$ & $\mathrm{R}$ & $\mathrm{R}$ & $\mathrm{R}$ & No $R$ & $\mathrm{R}$ & $\mathrm{R}$ & $\mathrm{R}$ \\
\hline Australia & No $R$ & & $\mathrm{R}$ & $\mathrm{R}$ & $\mathrm{R}$ & $\mathrm{R}$ & No $R$ & $\mathrm{R}$ & $\mathrm{R}$ & $\mathrm{R}$ \\
\hline Chile & $\mathrm{R}$ & $\mathrm{R}$ & & $\mathrm{R}$ & $\mathrm{R}$ & $\mathrm{R}$ & $\mathrm{R}$ & No $\mathrm{R}$ & $\mathrm{R}$ & $\mathrm{R}$ \\
\hline Dinamarca & $\mathrm{R}$ & $\mathrm{R}$ & $\mathrm{R}$ & & $\mathrm{R}$ & $\mathrm{R}$ & $\mathrm{R}$ & $\mathrm{R}$ & No $R$ & $\mathrm{R}$ \\
\hline España & $\mathrm{R}$ & $\mathrm{R}$ & $\mathrm{R}$ & $\mathrm{R}$ & & $\mathrm{R}$ & $\mathrm{R}$ & $\mathrm{R}$ & $\mathrm{R}$ & $\mathrm{R}$ \\
\hline Nicaragua & $\mathrm{R}$ & $\mathrm{R}$ & $\mathrm{R}$ & $\mathrm{R}$ & $\mathrm{R}$ & & $\mathrm{R}$ & $\mathrm{R}$ & $\mathrm{R}$ & $\mathrm{R}$ \\
\hline $\begin{array}{l}\text { Nueva } \\
\text { Zelanda }\end{array}$ & No $R$ & No $R$ & $\mathrm{R}$ & $\mathrm{R}$ & $\mathrm{R}$ & $\mathrm{R}$ & & $\mathrm{R}$ & $\mathrm{R}$ & $\mathrm{R}$ \\
\hline Perú & $\mathrm{R}$ & $\mathrm{R}$ & No $R$ & $\mathrm{R}$ & $\mathrm{R}$ & $\mathrm{R}$ & $\mathrm{R}$ & & $\mathrm{R}$ & No $R$ \\
\hline Turquía & $\mathrm{R}$ & $\mathrm{R}$ & $\mathrm{R}$ & No $R$ & $\mathrm{R}$ & $\mathrm{R}$ & $\mathrm{R}$ & $\mathrm{R}$ & & $\mathrm{R}$ \\
\hline Uruguay & $\mathrm{R}$ & $\mathrm{R}$ & $\mathrm{R}$ & $\mathrm{R}$ & $\mathrm{R}$ & $\mathrm{R}$ & $\mathrm{R}$ & No R & $\mathrm{R}$ & \\
\hline
\end{tabular}

Nota: $\mathrm{R}$ significa que se rechaza la hipótesis nula para el contraste entre esos países, mientras que NO $\mathrm{R}$ significa no rechazarla. La columna sombreada es En celeste se presentan las pruebas para el caso de Uruguay.

Fuente: Elaboración propia en base a Hidalgo et al. (2007) y Comtrade.

En primer lugar, al rechazar la hipótesis nula para la mayoría de los casos, se puede establecer que los resultados hallados para el estadístico $B F O$ son significativamente distintos, por lo que se concluye que las economías analizadas poseen diferentes potencialidades para el cambio estructural.

En segundo lugar los resultados parecen señalar algunos grupos de países para los cuales esta capacidad no es significativamente distinta. Son los casos de Australia con Nueva Zelanda, Dinamarca con Turquía, y Perú con Uruguay, para los cuales ambos contrastes no rechazan la hipótesis nula. El Contraste de Tukey, que como se señaló es más exigente para rechazar la hipótesis nula, agrega al 
primer grupo a Argentina que tendría un $B F O$ similar a ambos países, y agrega al tercer grupo a Chile que tendría un $B F O$ similar al de Perú, aunque no al de Uruguay.

En síntesis, se presenta evidencia significativa con respecto a las diferencias en las potencialidades para el cambio estructural de estas diez economías. Sin embargo, debe recordarse que el indicador $B F O$ incluye el aporte de bienes estratégicos que no son producidos por el país, por lo que el verdadero desempeño relativo de los países, en cuanto a su crecimiento económico, dependerá de en qué medida se logren alcanzar esos procesos productivos.

\subsection{Otros comentarios a resaltar a partir de los resultados obtenidos}

Es posible realizar, además, tres apreciaciones importantes a partir de los resultados anteriores. En primer lugar, el análisis realizado en el Capítulo 3 permite hacer una lectura del resultado del $B F O$ a la luz de la concentración de la canasta de exportaciones. En dicho capítulo, se ubica la diferencia entre países en el peso relativo de los diferentes rubros dentro de la canasta exportadora. Mientras que los países mejor posicionados en términos de $B F O$ presentan canastas bien diversificadas (como España, Turquía y Dinamarca), los países con menor $B F O$ promedio parecen tener exportaciones bastante más concentradas (como Chile, Perú, Uruguay y Nicaragua). Así, respecto a estos países con estructuras productivas basadas en Recursos Naturales la evidencia parece señalar que cuanto más diversificada es su producción más probable será que el país genere acumulaciones en diversos procesos productivos $\mathrm{y}$, por lo tanto, mejores serán sus oportunidades para el cambio estructural. En el mismo sentido, en Rodrik (2005) se plantea la importancia que posee la diversificación del patrón de producción en los procesos de desarrollo económico de los países.

En segundo lugar, el análisis realizado en el apartado 5.2 ayuda a visualizar mejor las diferentes maneras en que la potencialidad se puede manifestar, al presentar las diferentes formas que adoptan la frontera de eficiencia y los bienes estratégicos para cada país. Aquellos países con elevados valores de $B F O$ (España, Dinamarca y Turquía) poseen nubes de bienes no producidos mucho más cercanas a su producción actual y, a su vez, más homogéneamente dispuesta, al ser menor la diferencia entre los bienes que se encuentran cerca y los que no. Nuevamente, esto indicaría que estos países, al dedicarse a una producción mucho más diversa, han logrado acumular capacidades tecnológicas más variadas, por lo que encuentran relativamente más sencilla la producción de diferentes tipos de bienes.

Por último, países que presentan similares valores del estadístico $B F O$ que sintetiza las potencialidades para el cambio estructural, pueden tener por delante procesos de cambio estructural con trayectorias diferentes, como es el caso de Uruguay y Perú. En efecto, si bien para este par de países no se rechaza la hipótesis nula de igualdad de la media del estadístico $B F O$, por lo que se interpreta que 
ambos poseen las mismas potencialidades para el cambio estructural, un análisis de su ubicación en el Espacio del Producto y de los bienes estratégicos que enfrentan ambos países demuestra que el camino a recorrer por cada uno de ellos es diferente. Los gráficos siguientes (Gráficos 6) muestran la situación de Uruguay y Perú en el año 2006 tanto en la canasta de exportaciones como en los bienes estratégicos (recordar las referencias de la Figura 1).

\section{Gráficos 6}

Canasta de exportaciones de Uruguay y Perú y bienes estratégicos para el año 2006

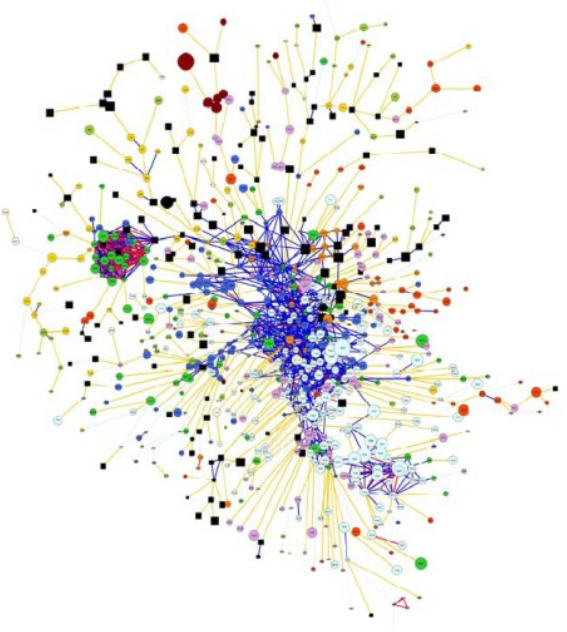

Canasta de Exportación de Uruguay 2006

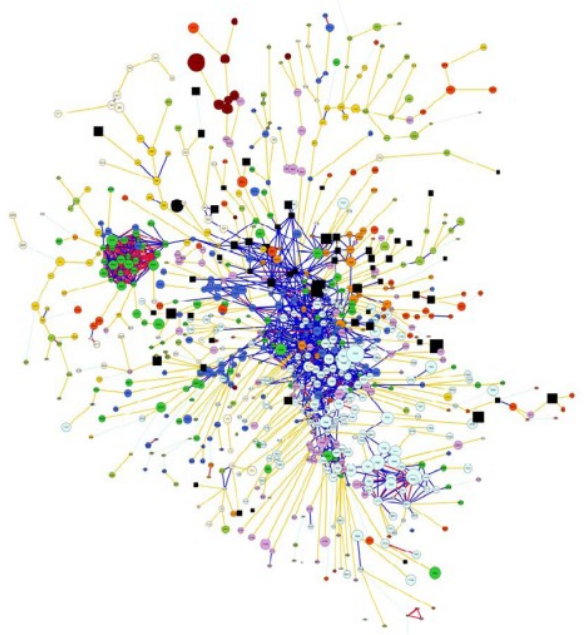

Canasta de Bienes Estratégicos Uruguay 2006

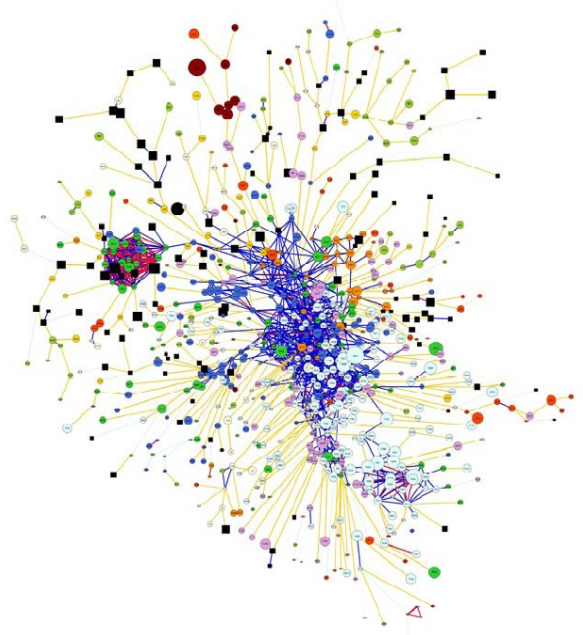

Canasta de Exportación de Perú 2006

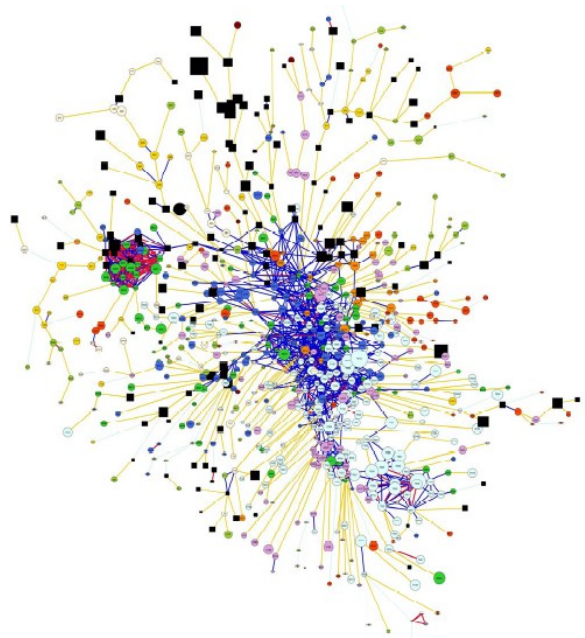

Canasta de Bienes Estratégicos Perú 2006

Nota: los cuadrados de color negro representan los bienes en los que los países presentan ventajas comparativas reveladas $(\mathrm{RCA}>1)$ en los gráficos de las canastas de exportación e indican los bienes estratégicos en los otros gráficos.

Elaboración propia en base a Hidalgo et al. (2007) y COMTRADE. 
La canasta de exportación de estos países muestra que ambos países ubican sus ventajas comparativas en la periferia del Espacio del Producto. Sin embargo, mientras que Uruguay concentra sus ventajas comparativas reveladas en la zona que corresponde a bienes forestales y bienes pecuarios, Perú lo hace en los bienes de la minería, la pesca y, en menor medida, la vestimenta. Resulta entonces notorio que estos países, a pesar de tener una potencialidad similar para el cambio estructural, se posicionan actualmente en lugares diferentes del Espacio del Producto. Esto reafirma la idea de que los procesos de cambio estructural no son lineales y parten desde diferentes realidades, aunque este punto debe ser estudiado más profundamente en futuros trabajos.

\section{Comentarios finales}

El presente trabajo ha discutido las potencialidades para llevar a cabo un cambio estructural que promueva y acompañe la convergencia de ingresos para un conjunto de países con especializaciones productivas similares. El trabajo utilizó las herramientas analíticas del Espacio del Producto presentado por Hausmann y otros, enriqueciendo su lectura a través de la tradición y las ideas más recientes de la CEPAL.

A partir de las pruebas de significación presentadas, es posible afirmar que los países seleccionados presentan diferentes potencialidades para el cambio estructural. Las potencialidades de Uruguay, son sólo asimilables a las de Perú. España, Dinamarca, Turquía, Argentina, Nueva Zelanda, y Australia presentan mayores potencialidades y Chile y Nicaragua menores.

Otra conclusión central del trabajo es que para estos países basados en recursos naturales, mayores potencialidades para el cambio estructural se relacionan con una canasta de exportación más diversificada, lo que confirma la idea de que la diversificación productiva promueve un aprendizaje tecnológico más amplio y, por lo tanto, ensancha las potencialidades de modificación estructural. Al extraerse esta conclusión debe recordarse que el marco analítico que se utilizó aquí considera únicamente datos de exportaciones, ya que estos conforman el patrón de especialización, no de toda la estructura productiva de una economía.

El herramental analítico utilizado es compatible con la afirmación teórica de que el proceso de cambio estructural es no lineal. Se entiende que este herramental supera limitaciones de los marcos analíticos anteriores, como el Modelo Ricardiano de Heterogeneidad Estructural que supone un proceso de cambio estructural lineal. Así, países con similares potencialidades pueden tener, por delante, procesos de cambio estructural basados en diferentes tipos de bienes, como es el caso de la comparación entre Uruguay y Perú. Además el marco del 
Espacio del Producto permite señalar que, si la meta es converger en ingresos, la estrategia productiva adecuada no es aquélla que intenta agregar más valor a los bienes ya exportados con ventajas comparativas por una economía, sino que debe ser una estrategia que promueva el cambio estructural.

Por último se debe aclarar que el hecho de que algunos países tengan menos potencialidades para el cambio estructural no necesariamente implica que los países estén condenados al rezago en este sentido, sino que el esfuerzo gubernamental deberá ser mayor, tanto para alcanzar los bienes considerados estratégicos actualmente, como para explorar políticas que intenten desarrollar nuevas capacidades más allá de éstos. Debe recordarse que el objetivo de las políticas de desarrollo productivo es aprovechar las capacidades tecnológicas para inducir a los privados a la generación de innovaciones, las cuales incluyen la producción de nuevos productos. Sin embargo, dado que el cambio estructural es un proceso no lineal, es particularmente importante en el caso de estos países, que la política de desarrollo productivo induzca al desarrollo de capacidades tecnológicas estratégicas en nuevas tecnologías transversales. Seguramente, éstas resulten claves para el logro del cambio estructural en el futuro y, por lo tanto, para la convergencia de ingresos.

\section{Bibliografía}

AGHION, P.; HOWIT, P. A model of growth through creative destruction. Econometrica, 60, p. 323-351, 1992.

ÁlVAREZ, J.; BÉRTOLA, L.; PORCILE, G. Primos ricos y empobrecidos: crecimiento, distribución del ingreso e instituciones en Australia-Nueva Zelanda vs Argentina-Uruguay. Editorial Fin de Siglo, 2007.

BALASSA, B. Comparative advantage in manufactured goods: a reappraisal. The Review of Economics and Statistics, v. 68, n. 2, p. 315-319, 1986.

BÉRTOLA, L. Ensayos de historia económica: Uruguay en la región y el mundo. Ediciones Trilce, 2000.

BITTENCOURT, G. Imaginando la economía uruguaya en las próximas dos décadas. Montevideo: Universidad de la República. Departamento de Economía, Facultad de Ciencias Sociales, 2003. (DT 16/03).

BRUNINI, A.; FLEITAS, S.; OURENS, G. Crecimiento económico y especialización productiva. Los caminos de la transformación estructural de Uruguay en comparación con otras economías intensivas en recursos naturales (1985-2006). Tesis (Monografía) Facultad de Ciencias Económicas y de Administración de la Universidad de la República, Montevideo, Uruguay, 2009.

BURGUEÑO, O.; PITTALUGA, L. El enfoque neo-shumpeteriano de la tecnología. Revista Quantum, v. 1, n. 3, p. 5-32, 1994. 
CEPAL. La transformación productiva 20 años después. Viejos problemas, nuevas oportunidades. Santiago de Chile: Naciones Unidas, 2008.

Progreso técnico y cambio estructural en América Latina. Santiago de Chile: Naciones Unidas-IDRC, 2007.

. Desarrollo productivo en economías abiertas. Santiago de Chile: Naciones Unidas, 2004.

Globalización y desarrollo. Santiago de Chile: Naciones Unidas, 2002.

Equidad, desarrollo y ciudadanía. Santiago de Chile: Naciones Unidas, 2000.

CIMOLI, M. Heterogeneidad estructural, asimetrías tecnológicas y crecimiento de América Latina. Santiago de Chile: Naciones Unidas, 2005.

Technological gaps and institutional asymmetries in a north-south model with a continuum of goods. Metroeconómica, n. 39, p. 245-274, 1988.

; PORCILE, G. Volatility and crisis in catching-up economies industrial paththrough under the stickiness of technological capabilities and 'The Red Queen Effect'. In: RAFI KHAN, S.; CHRISTIANSEN, J. Towards new developmentalism market as means rather than master. Routledge, 2008.

; ROVIRA, S. Structural change and the balance-of-payments constraint: why did Latin America fail to converge? Cambridge Journal of Economics, 2008 .

; SOETE, L. A generalized technology gap trade model. Economie Appliquée, tome XLV, n. 3, p. 33-54, 1992.

DOSI, G. Technical paradigms and technological trajectories: a suggested interpretation of the determinants of technical change. Research Policy, v. II, n. 3, p. 147-162, 1982.

FAJNZYLBER, F. [1990]. Industrialización en América Latina: de la "caja negra" al “casillero vacío. Nueva Sociedad, n. 118, p. 21-28, Mar./Abr. 1992.

FEENSTRA, R.; LIPSEY, R.; DENG, H.; MA, A.; MO, H. World trade flows: 1962-2000. Cambridge, MA: National Bureau of Economic Research, 2005. (NBER Working Paper, 11040).

GROSSMAN, G.; HELPMAN, E. Innovation and growth in the global economy. Cambridge: MIT Press, 1991.

HAUSMANN, R. ¿Cómo crecen los países? ¿Cómo puede crecer Colombia? Ponencia presentada en la XLII Convención Bancaria, Cartagena, 21 de junio de 2007.

; HWANG, J.; RODRIK, D. What you export matters. Harvard University, 2005. (CID Working Paper, 123).

; KLINGER, B. Achieving export led growth in Colombia. Harvard University. 2008. (CID Working Paper, 182). 
HAUSMANN, R.; KLINGER, B. Growth diagnostic: Perú. Harvard University, 2007. (CID Working Paper, CSI-111).

University, 2007. . Growth diagnostic: Belize. CID preparado para el BID. Harvard ; $\quad$. The structure of the product space and the evolution of comparative advantage. Harvard University, 2007. (CID Working Paper, 146).

Working Paper). . Growth diagnostic: Paraguay. Harvard University, 2007. (CID (CID Working Paper, 129).

. South Africa's export predicament. Harvard University, 2006. ; . Structural transformation and patterns of comparative advantage in the product space. Harvard University, 2006. (CID Working Paper, 128). ; ; LAWRANCE, R. Examining beneficiation. Harvard University, 2008. (CID Working Paper, 162).

; RODRIGUEZ-CLARE, A.; RODRIK, D. Towards a strategy for economic growth in Uruguay. BID, Economic and Social Study Series, RE1-05-003, 2005.

; RODRIK, D. Doomed to choose: industrial policy as predicament. John F. Kennedy School of Government, Harvard University, 2006.

; ___ SABEL, C. Reconfiguring industrial policy. Harvard University, 2007. (CID Working Paper, 168).

HIDALGO, C.; KLINGER, B.; BARABASI, A.; HAUSMANN, R. The product space conditions the development of nations. Science, p. 482-487, 2007.

HOLLAND, M.; PORCILE. G. Brecha tecnológica y crecimiento en América Latina. CEPAL BID, 2005.

KATZ, J.; STUMPO, G. Regímenes sectoriales, productividad y competitividad internacional. Revista de la CEPAL, n. 75 (LC/G.2150-P/E), dic. 2001.

LEAMER, E. Sources of comparative advantage: theory and evidence. Cambridge MA: The MIT Press, 1984.

LÓPEZ, A. Las ideas evolucionistas en economía: una visión de conjunto. Documentos de trabajo del PHES de la Facultad de Ciencias Sociales de la UdelaR, Uruguay, 1996.

MONDELLI, F.; RODRÍGUEZ, M. Patrones de especialización tecnológica en el comercio exterior de América Latina y OCDE (1986-2005). Trabajo de investigación monográfica, Facultad de Ciencias Económicas y de Administración UdelaR, Uruguay, 2008 .

NELSON, R.; WINTER, S. An evolutionary theory of economic growth. Cambridge Univesity Press, 1982. 
PITTALUGA, L. Una visión de la economía: la innovación tecnológica como motor de transformación de las estructuras productivas. Ciencia, Tecnología y Sociedad, Montevideo, 2008.

PNUD. Desarrollo humano en Uruguay 2005: el Uruguay hacia una estrategia de desarrollo basada en el conocimiento. In: PNUD. Informe Nacional de Desarrollo Humano2005. Montevideo: PNUD-Uruguay, Naciones Unidas, 2005.

PNUD. Desarrollo humano en Uruguay 2008: política, políticas y desarrollo humano. In: PNUD. Informe Nacional de Desarrollo Humano-2008. Montevideo: PNUD-Uruguay, Naciones Unidas, 2008.

PORCILE, G.; HOLLAND, M.; CIMOLI, M.; ROSAS, L. Especialización, tecnología y crecimiento en el modelo Ricardiano. Nova Economia, Belo Horizonte, v. 16, n. 3, p. 483 $506,2006$.

RODRIK, D. Políticas de diversificación económica. Revista de la CEPAL, 87, 2005.

ROMER, P. Increasing returns and long-run growth. Journal of Political Economy, University of Chicago Press, v. 94, n. 5, p. 1002-1037, 1986. 